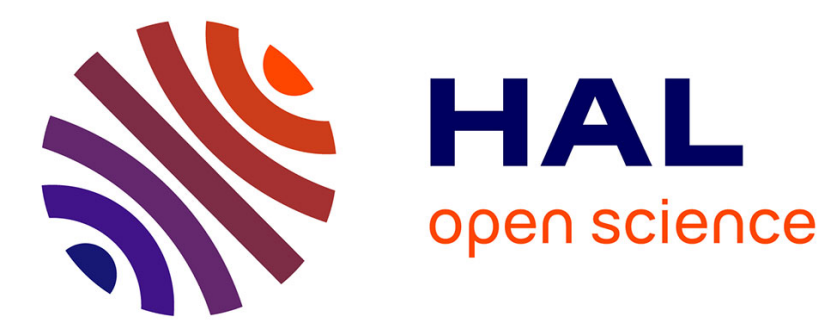

\title{
The gauge theory of dislocations: conservation and balance laws
}

\author{
Markus Lazar, Charalampos Anastassiadis
}

\section{To cite this version:}

Markus Lazar, Charalampos Anastassiadis. The gauge theory of dislocations: conservation and balance laws. Philosophical Magazine, 2008, 88 (11), pp.1673-1699. 10.1080/14786430802255653 . hal00513919

\section{HAL Id: hal-00513919 \\ https://hal.science/hal-00513919}

Submitted on 1 Sep 2010

HAL is a multi-disciplinary open access archive for the deposit and dissemination of scientific research documents, whether they are published or not. The documents may come from teaching and research institutions in France or abroad, or from public or private research centers.
L'archive ouverte pluridisciplinaire HAL, est destinée au dépôt et à la diffusion de documents scientifiques de niveau recherche, publiés ou non, émanant des établissements d'enseignement et de recherche français ou étrangers, des laboratoires publics ou privés. 


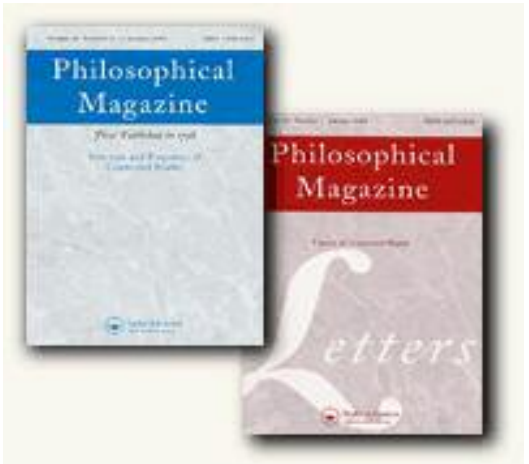

The gauge theory of dislocations: conservation and balance laws

\begin{tabular}{|r|l|}
\hline Journal: & Philosophical Magazine \& Philosophical Magazine Letters \\
\hline Manuscript ID: & TPHM-08-Mar-0091.R1 \\
\hline Journal Selection: & Philosophical Magazine \\
\hline Date Submitted by the & 03-Jun-2008 \\
\hline Complete List of Authors: & $\begin{array}{l}\text { Lazar, Markus; Institute of Condensed Matter Physics, Darmstadt } \\
\text { University of Technology } \\
\text { Knastassiadis, Charalampos; TUD }\end{array}$ \\
\hline Keywords: & defects in solids, dislocation theory, mechanics \\
\hline Keywords (user supplied): & conservation laws \\
\hline \hline $\begin{array}{l}\text { Note: The following files were submitted by the author for peer review, but cannot be converted } \\
\text { to PDF. You must view these files (e.g. movies) online. }\end{array}$ \\
\hline \begin{tabular}{l} 
Lazar disloc1b.tex \\
\hline
\end{tabular}
\end{tabular}

\section{S) ScholaroNE Manuscript Central}




\title{
The gauge theory of dislocations: conservation and balance laws
}

\author{
Markus Lazar *, Charalampos Anastassiadis \\ Emmy Noether Research Group, \\ Department of Physics, \\ Darmstadt University of Technology, \\ Hochschulstr. 6, \\ D-64289 Darmstadt, Germany
}

June 3, 2008

\begin{abstract}
We derive conservation and balance laws for the translational gauge theory of dislocations by applying Noether's theorem. We present an improved translational gauge theory of dislocations including the dislocation density tensor and the dislocation current tensor. The invariance of the variational principle under the continuous group of transformations is studied. Through Lie's-infinitesimal invariance criterion we obtain conserved translational and rotational currents for the total Lagrangian made up of an elastic and dislocation part. We calculate the broken scaling current. Looking only on one part of the whole system, the conservation laws are changed into balance laws. Because of the lack of translational, rotational and dilatation invariance for each part, a configurational force, moment and power appears. The corresponding $\boldsymbol{J}, \boldsymbol{L}$ and $M$ integrals are obtained. Only isotropic and homogeneous materials are considered and we restrict ourselves to a linear theory. We choose constitutive laws for the most general linear form of material isotropy. Also we give the conservation and balance laws corresponding to the gauge symmetry and the addition of solutions. From the addition of solutions we derive a reciprocity theorem for the gauge theory of dislocations. Also, we derive the conservation laws for stress-free states of dislocations.
\end{abstract}

Keywords: Conservation laws; balance laws; Noether symmetries; dislocations; gauge theory.

${ }^{*}$ Corresponding author. E-mail address: lazar@fkp.tu-darmstadt.de (M. Lazar). 


\section{Introduction}

In the Lagrangian field theory of elasticity the existence of inhomogeneities in an elastic medium is expressed through the explicit dependence of the Lagrangian on the position. Due to different positions taking up from the inhomogeneity in the material a change in the potential and kinetic energy will take place. This shows up as a configurational force acting on the inhomogeneity being an elastic singularity. Understanding elasticity as a classical field theory, Eshelby [1, 2] and Morse and Feshbach [3] derived the elastic energy-momentum tensor, called also Eshelby stress tensor. Supplementary, Eshelby [1] and Morse and Feshbach [3] introduced independently the field intensity vector and the field momentum vector of elasticity.

Later Günther [4] used the Noether theorem to find all the continuous transformation groups leaving the potential energy density of linear elastostatics invariant. With the known Lie-point symmetries he constructed conserved currents and path-independent integrals and related them to torsion and bending problems of rods, plates and shells. Knowles and Sternberg [5] extended these results for the case of finite elasticity. Fletcher [6] continued further and calculated the variational symmetries and conserved integrals in linear elastodynamics. Moreover, Olver [7] investigated the Lie-point and Lie-Bäcklund symmetries of elastostatics and derived the corresponding conservation laws. An overview of conservation and balance laws of elasticity can be found in the books of Maugin [8] and Kienzler and Herrmann [9].

In the gauge theory of classical electrodynamics the inhomogeneous Maxwell equations can be obtained from a Lagrangian density after varying the scalar and vector gauge potentials. The Lagrangian density consists of two parts [10, 11], describing the electromagnetic field and another one being responsible for the interaction between the electromagnetic field and the charge and current density. Kadić and Edelen [12] proposed a theory of dislocations in a continuum with gauge invariant equations of motion. The formal structure of these equations is analoque to the inhomogeneous Maxwell-equations. Kadić and Edelen [13] and Edelen and Lagoudas [14] presented a mathematical gauge theory of Yang-Mills type for elasticity including dislocations. They derived equations of motions and suggested some improvement since the given constitutive laws were inadequately chosen. In general the formal structure of the theory was correct, since canonical energy-momentum tensors and configurational forces such as the Peach-Koehler force [15] has been successfully calculated.

In this paper we give a complete system of equations of motion for the translational gauge theory of dislocations. We calculate the Lie-point symmetries of this system and also the variational and divergence symmetries of the Lagrangian, following the standard technique of Lie-group analysis given in the book of Olver [16]. The canonical energymomentum and angular-momentum tensors, as well as the dilatation current vector are determined. The generalization of the conserved integrals from the linear theory of elastodynamics to a translational gauge theory of dislocations is given. Symmetry-breaking follows by taking into account only the elastic or dislocation part of the system. In this case we calculate the configurational force, moment and power acting on dislocations. 


\section{Gauge theory of elasticity}

According to the field theory of elasticity, the equations of motion for a compatible continuum can be derived from a variational principle with the Lagrangian of linear elasticity

$$
\mathcal{L}_{\mathrm{e}}=T_{\mathrm{e}}-W_{\mathrm{e}}=\frac{1}{2} p_{i} \dot{u}_{i}-\frac{1}{2} \sigma_{i j} u_{i, j}
$$

The canonical conjugate quantities to the kinematical velocity $\dot{u}_{i}$ and displacement gradient $u_{i, j}$ are thereby defined as follows

$$
p_{i}:=\frac{\partial \mathcal{L}_{\mathrm{e}}}{\partial \dot{u}_{i}}, \quad \sigma_{i j}:=-\frac{\partial \mathcal{L}_{\mathrm{e}}}{\partial u_{i, j}}
$$

These are the momentum vector $p_{i}$ and the force stress tensor $\sigma_{i j}$. Since the works of Kadic and Edelen [12,13] and Edelen and Lagoudas [14] it is known that symmetry breaking of the homogeneity of the action of the three-dimensional translational group $T(3)$ in elasticity leads to a gauge theory of dislocations. In terms of symmetry, we require from the Lagrangian density (2.1) to stay invariant under the internal transformation of the displacement field

$$
u_{i}^{\prime}=u_{i}-f_{i}
$$

given with an arbitrary vector-function $f_{i}(t, x)$. The transformation $(2.3)$ represents the generalization of a rigid body translation with $f_{i}=$ const. This requirement can only be fulfilled by introducing the gauge potentials $\varphi$ and $\phi$. They restore the invariance of (2.1) through the following two steps. First of all, a minimal replacement

$$
\beta_{i j}:=\nabla_{j} u_{i}=u_{i, j}+\phi_{i j}, \quad v_{i}:=\nabla_{t} u_{i}=\dot{u}_{i}+\varphi_{i},
$$

of the displacement gradient $u_{i, j}$ and Newtonian kinematic velocity $\dot{u}_{i}$ by the translational gauge-covariant derivatives $\nabla_{j} u_{i}$ and $\nabla_{t} u_{i}$ is used. These represent the elastic distortion tensor $\beta_{i j}$ and elastic velocity vector $v_{i}$, respectively. Both quantities are now incompatible. The gauge potentials may be identified with the plastic velocity and plastic distortion: $\varphi_{i}=-v_{i}^{\mathrm{P}}$ and $\phi_{i j}=-\beta_{i j}^{\mathrm{P}}$. On the other hand, for compensating the time $\dot{f}_{i}$ and space $f_{i, j}$ derivatives in the Lagrangian (2.1) caused by (2.3) the gauge fields have to transform as follows

$$
\varphi_{i}^{\prime}=\varphi_{i}+\dot{f}_{i}, \quad \phi_{i j}^{\prime}=\phi_{i j}+f_{i, j}
$$

The transformations (2.5) and (2.3) are making up the local gauge transformations leaving the Lagrangian density

$$
\mathcal{L}_{\mathrm{e}}=\frac{1}{2} p_{i} v_{i}-\frac{1}{2} \sigma_{i j} \beta_{i j}
$$

form-invariant called gauge-invariant. The elastic Lagrangian density (2.6) describes an incompatible elastic material. By construction a local gauge symmetry is valid

$$
v_{i}^{\prime}=v_{i}, \quad \beta_{i j}^{\prime}=\beta_{i j} .
$$


Dislocations being now present contribute also by themselves to the total energy of the system. With the gauge fields $\varphi$ and $\phi$ we can introduce the two translational field strengths

$$
T_{i j k}=\phi_{i k, j}-\phi_{i j, k}, \quad I_{i j}=-\varphi_{i, j}+\dot{\phi}_{i j}
$$

or in terms of $\beta_{i j}$ and $v_{i}$

$$
T_{i j k}=\beta_{i k, j}-\beta_{i j, k}, \quad I_{i j}=-v_{i, j}+\dot{\beta}_{i j}
$$

called the dislocation tensor (torsion tensor) and the dislocation current tensor, respectively. These are the kinematical quantities of dislocations. They are related to each other through the Bianchi identities

$$
\epsilon_{j k l} T_{i j k, l}=0, \quad \dot{T}_{i j k}+2 I_{i[j, k]}=0 .
$$

The transformation of the fields $\left(u_{i}, \varphi_{i}, \phi_{i j}\right)$ according to the local gauge transformations (2.5) and (2.3) leaves the quantities

$$
T_{i j k}^{\prime}=T_{i j k}, \quad I_{i j}^{\prime}=I_{i j},
$$

gauge-invariant. The torsion tensor $T_{i j k}$ was introduced by Élie Cartan [17] as a generalization from Riemannian to non-Riemannian geometry. Kondo [18, 19], Bilby et al. [20, 21] and Kröner [22] understood the meaning of the three-dimensional torsion tensor as the dislocation density tensor. The Lagrangian of the dislocation field can be written in the following form

$$
\mathcal{L}_{\mathrm{di}}=T_{\mathrm{di}}-W_{\mathrm{di}}=\frac{1}{2} D_{i j} I_{i j}-\frac{1}{4} H_{i j k} T_{i j k},
$$

where $T_{\mathrm{di}}$ and $W_{\mathrm{di}}$ describe the kinetic and potential energy density of dislocations. The total Lagrangian density describing the whole system (in linear approximation) is given as the sum of (2.6) and (2.12)

$$
\mathcal{L}=T-W=\mathcal{L}_{\mathrm{e}}+\mathcal{L}_{\mathrm{di}}=\frac{1}{2} p_{i} v_{i}-\frac{1}{2} \sigma_{i j} \beta_{i j}+\frac{1}{2} D_{i j} I_{i j}-\frac{1}{4} H_{i j k} T_{i j k} .
$$

The canonical conjugate quantities can be obtained from $\mathcal{L}$ as

$$
p_{i}:=\frac{\partial \mathcal{L}}{\partial v_{i}}, \quad \sigma_{i j}:=-\frac{\partial \mathcal{L}}{\partial \beta_{i j}}, \quad D_{i j}:=\frac{\partial \mathcal{L}}{\partial I_{i j}}, \quad H_{i j k}:=-2 \frac{\partial \mathcal{L}}{\partial T_{i j k}},
$$

$p_{i}, \sigma_{i j}, I_{i j}$, and $H_{i j k}$ are the momentum vector, the force stress tensor, the dislocation momentum flux tensor, and the pseudomoment stress tensor, respectively.

The Euler-Lagrange equations derived from the total Lagrangian $\mathcal{L}=\mathcal{L}\left(v_{i}, \beta_{i j}, I_{i j}, T_{i j k}\right)$ are given by

$$
\begin{aligned}
& E_{i}^{u}(\mathcal{L})=\mathrm{D}_{t} \frac{\partial \mathcal{L}}{\partial \dot{u}_{i}}+\mathrm{D}_{j} \frac{\partial \mathcal{L}}{\partial u_{i, j}}=0 \\
& E_{i}^{\varphi}(\mathcal{L})=\mathrm{D}_{t} \frac{\partial \mathcal{L}}{\partial \dot{\varphi}_{i}}+\mathrm{D}_{j} \frac{\partial \mathcal{L}}{\partial \varphi_{i, j}}-\frac{\partial \mathcal{L}}{\partial \varphi_{i}}=0 \\
& E_{i j}^{\phi}(\mathcal{L})=\mathrm{D}_{t} \frac{\partial \mathcal{L}}{\partial \dot{\phi}_{i j}}+\mathrm{D}_{k} \frac{\partial \mathcal{L}}{\partial \phi_{i j, k}}-\frac{\partial \mathcal{L}}{\partial \phi_{i j}}=0,
\end{aligned}
$$


where $\mathrm{D}_{t}$ and $\mathrm{D}_{i}$ are thereby the so-called total derivatives:

$$
\begin{aligned}
& \mathrm{D}_{t}=\frac{\partial}{\partial t}+\dot{u}_{\alpha} \frac{\partial}{\partial u_{\alpha}}+\dot{\varphi}_{\alpha} \frac{\partial}{\partial \varphi_{\alpha}}+\dot{\phi}_{\alpha} \frac{\partial}{\partial \phi_{\alpha}}+\ldots \\
& \mathrm{D}_{i}=\frac{\partial}{\partial x_{i}}+u_{\alpha, i} \frac{\partial}{\partial u_{\alpha}}+u_{\alpha, i j} \frac{\partial}{\partial u_{\alpha, j}}+\varphi_{\alpha, i j} \frac{\partial}{\partial \varphi_{\alpha, j}}+\phi_{\alpha, i j} \frac{\partial}{\partial \phi_{\alpha, j}}+\ldots
\end{aligned}
$$

Written in terms of the canonical conjugate quantities (2.14), Eqs. (2.15)-(2.17) take the form

$$
\begin{aligned}
\mathrm{D}_{t} p_{i}-\mathrm{D}_{j} \sigma_{i j} & =0 \\
\mathrm{D}_{j} D_{i j}+p_{i} & =0 \\
\mathrm{D}_{t} D_{i j}+\mathrm{D}_{k} H_{i j k}+\sigma_{i j} & =0 .
\end{aligned}
$$

Eq. (2.20) represents the balance of linear momentum. Eqs. (2.21) and (2.22) are the equations for the balance of dislocations. The coupled system (2.20)-(2.22) together with the Bianchi identities (2.10) are analogous to the inhomogeneous Maxwell equations. Like in electromagnetic field theory by coupling the field to external charges and currents $(\rho, \boldsymbol{j})$, the dislocation field will be influenced by the momentum and stresses $(\boldsymbol{p}, \boldsymbol{\sigma})$. The force stress $\boldsymbol{\sigma}$ and linear momentum $\boldsymbol{p}$ works like sources driving dislocations. The elastic medium plays also the role of transmitting the interaction like an elastic aether. The conservation of linear momentum appears as an integrability condition for the balance of dislocation equations. This can be seen by applying $\mathrm{D}_{t}$ on $(2.21)$ and $\mathrm{D}_{j}$ on $(2.22)$ and subtracting the last from the first one. It plays the same role like the conservation for the charge in the electromagnetic field theory. The most general linear constitutive equations for the momentum, the asymmetric force stress, the dislocation momentum flux tensor and the pseudomoment stress of an isotropic and centrosymmetric medium are

$$
\begin{aligned}
p_{i} & =\rho v_{i}, \\
\sigma_{i j} & =\lambda \delta_{i j} \beta_{k k}+\mu\left(\beta_{i j}+\beta_{j i}\right)+\gamma\left(\beta_{i j}-\beta_{j i}\right), \\
D_{i j} & =d_{1} \delta_{i j} I_{k k}+d_{2}\left(I_{i j}+I_{j i}\right)+d_{3}\left(I_{i j}-I_{j i}\right), \\
H_{i j k} & =c_{1} T_{i j k}+c_{2}\left(T_{j k i}+T_{k i j}\right)+c_{3}\left(\delta_{i j} T_{l l k}+\delta_{i k} T_{l j l}\right),
\end{aligned}
$$

where $\rho$ is the mass density and with 9 material constants $\mu, \nu, \gamma, c_{1}, \ldots, c_{3}$ and $d_{1}, \ldots, d_{3}$. Here we have used a constitutive relation for the dislocation momentum flux tensor $D_{i j}=L_{i j k l} I_{k l}$ with $L_{i j k l}=L_{k l i j}$. A similar constitutive law was used for material with microstructure by Maugin [23]. Maugin [23] also noted that for the isotropic microinertia is often assumed a too simple form which is not imposed by the formulation of the theory. In Eq. (2.25) we have assumed the most general one for an isotropic theory. On the other hand, Kadić and Edelen [13] and Edelen and Lagoudas [14] used the constitutive relation $D_{i j}=s_{2} I_{i j}$ which is to simple.

The requirement of non-negativity of the energy (material stability) $E=T+W \geq 0$ leads to the conditions of positive semidefiniteness of the material constants. Since $v_{i}$ $\beta_{i j}, I_{i j}$ and $T_{i j k}$ are uncoupled from each other, the conditions can be studied separately: $T_{\mathrm{e}} \geq 0, T_{\mathrm{di}} \geq 0, W_{\mathrm{e}} \geq 0$ and $W_{\mathrm{di}} \geq 0$. Thus, the characteristic constants of the material 
have to satisfy the following conditions

$$
\begin{aligned}
& \rho \geq 0 \\
& d_{2} \geq 0, \quad d_{3} \geq 0, \quad 3 d_{1}+2 d_{2} \geq 0, \\
& \mu \geq 0, \quad \gamma \geq 0, \quad 3 \lambda+2 \mu \geq 0, \\
& c_{1}-c_{2} \geq 0, \quad c_{1}+2 c_{2} \geq 0, \quad c_{1}-c_{2}+2 c_{3} \geq 0 \text {. }
\end{aligned}
$$

Substituting the constitutive equations in the above system (2.20)-(2.22), we get for the field variables $u_{i}, \phi_{i j}, \varphi_{i}$ a system of 15 coupled linear partial differential equations $\Delta \equiv\left(\Delta_{1}, \ldots, \Delta_{15}\right)=0$ :

$$
\begin{aligned}
& \rho\left(\ddot{u}_{i}+\dot{\varphi}_{i}\right)-\lambda\left(u_{j, j i}+\phi_{j j, i}\right)-(\mu+\gamma)\left(u_{i, j j}+\phi_{i j, j}\right)-(\mu-\gamma)\left(u_{j, i j}+\phi_{j i, j}\right)=0, \\
& d_{1}\left(\varphi_{j, j i}-\dot{\phi}_{j j, i}\right)+\left(d_{2}+d_{3}\right)\left(\varphi_{i, j j}-\dot{\phi}_{i j, j}\right)+\left(d_{2}-d_{3}\right)\left(\varphi_{j, i j}-\dot{\phi}_{j i, j}\right)-\rho\left(u_{i}+\varphi_{i}\right)=0 \\
& d_{1} \delta_{i j}\left(\dot{\varphi}_{k, k}-\ddot{\phi}_{k k}\right)+\left(d_{2}+d_{3}\right)\left(\dot{\varphi}_{i, j}-\ddot{\phi}_{i j}\right)+\left(d_{2}-d_{3}\right)\left(\dot{\varphi}_{j, i}-\ddot{\phi}_{j i}\right) \\
& \quad-c_{1}\left(\phi_{i k, j k}-\phi_{i j, k k}\right)-c_{2}\left(\phi_{j i, k k}-\phi_{j k, i k}+\phi_{k j, i k}-\phi_{k i, j k}\right)-c_{3}\left[\delta_{i j}\left(\phi_{l k, l k}-\phi_{l l, k k}\right)\right. \\
& \left.\quad+\left(\phi_{k k, j i}-\phi_{k j, k i}\right)\right]-\lambda \delta_{i j}\left(u_{k, k}+\phi_{k k}\right)-(\mu+\gamma)\left(u_{i, j}+\phi_{i j}\right)-(\mu-\gamma)\left(u_{j, i}+\phi_{j i}\right)=0 .
\end{aligned}
$$

Eq. (2.28) is a generalized inhomogeneous Navier equation for $u$. Eq. (2.29) has the form of a generalized inhomogeneous Helmholtz equation for $\varphi$ and Eq. (2.30) is a kind of generalization of an inhomogeneous Klein-Gordon equation for $\phi$. Due to the inhomogeneous parts they are coupled.

\section{Lie symmetries}

The infinitesimal continuous transformation acting on the independent $(t, \boldsymbol{x})$ and dependent $(\boldsymbol{u}, \boldsymbol{\varphi}, \boldsymbol{\phi})$ variables build a Lie group $G$. If $G$ is the Lie group of invariance of the system (2.20)-(2.22), then it is also a symmetry group. The infinitesimal group action for the independent and dependent variables has the form

$$
\begin{aligned}
x_{i}^{\prime} & =x_{i}+\varepsilon X_{i}(\boldsymbol{x}, t, \boldsymbol{u}, \boldsymbol{\varphi}, \boldsymbol{\phi})+\cdots \\
t^{\prime} & =t+\varepsilon \tau(\boldsymbol{x}, t, \boldsymbol{u}, \boldsymbol{\varphi}, \boldsymbol{\phi})+\cdots \\
u_{\alpha}^{\prime} & =u_{\alpha}+\varepsilon U_{\alpha}(\boldsymbol{x}, t, \boldsymbol{u}, \boldsymbol{\varphi}, \boldsymbol{\phi})+\cdots \\
\varphi_{\alpha}^{\prime} & =\varphi_{\alpha}+\varepsilon \Psi_{\alpha}(\boldsymbol{x}, t, \boldsymbol{u}, \boldsymbol{\varphi}, \boldsymbol{\phi})+\cdots \\
\phi_{\alpha \beta}^{\prime} & =\phi_{\alpha \beta}+\varepsilon \Phi_{\alpha \beta}(\boldsymbol{x}, t, \boldsymbol{u}, \boldsymbol{\varphi}, \boldsymbol{\phi})+\cdots
\end{aligned}
$$


where the infinitesimal generators are defined by

$$
\begin{aligned}
X_{i}(\boldsymbol{x}, t, \boldsymbol{u}, \boldsymbol{\varphi}, \boldsymbol{\phi}) & :=\left.\frac{\partial x_{i}^{\prime}}{\partial \boldsymbol{\varepsilon}}\right|_{\varepsilon=0}, \\
\tau(\boldsymbol{x}, t, \boldsymbol{u}, \boldsymbol{\varphi}, \boldsymbol{\phi}) & :=\left.\frac{\partial t^{\prime}}{\partial \varepsilon}\right|_{\varepsilon=0}, \\
U_{\alpha}(\boldsymbol{x}, t, \boldsymbol{u}, \boldsymbol{\varphi}, \boldsymbol{\phi}) & :=\left.\frac{\partial u_{\alpha}^{\prime}}{\partial \varepsilon}\right|_{\varepsilon=0}, \\
\Psi_{\alpha}(\boldsymbol{x}, t, \boldsymbol{u}, \boldsymbol{\varphi}, \boldsymbol{\phi}) & =\left.\frac{\partial \varphi_{\alpha}^{\prime}}{\partial \varepsilon}\right|_{\varepsilon=0}, \\
\Phi_{\alpha \beta}(\boldsymbol{x}, t, \boldsymbol{u}, \boldsymbol{\varphi}, \boldsymbol{\phi}) & =\left.\frac{\partial \phi_{\alpha \beta}^{\prime}}{\partial \boldsymbol{\varepsilon}}\right|_{\varepsilon=0} .
\end{aligned}
$$

These infinitesimal generators build the following vector field:

$$
\boldsymbol{\nu}=\tau \frac{\partial}{\partial t}+X_{i} \frac{\partial}{\partial x_{i}}+U_{\alpha} \frac{\partial}{\partial u_{\alpha}}+\Psi_{\alpha} \frac{\partial}{\partial \varphi_{\alpha}}+\Phi_{\alpha \beta} \frac{\partial}{\partial \phi_{\alpha \beta}} .
$$

Since the system (2.28)-(2.30) is of second order, we need the prolonged vector field of second order

$$
\operatorname{pr}^{(2)} \boldsymbol{\nu}=\boldsymbol{\nu}+\boldsymbol{a}+\boldsymbol{b}+\boldsymbol{c}
$$

where the vector fields $\boldsymbol{a}, \boldsymbol{b}, \boldsymbol{c}$ read

$$
\begin{aligned}
& \boldsymbol{a}=\bar{U}_{\alpha i} \frac{\partial}{\partial u_{\alpha, i}}+\bar{U}_{\alpha t} \frac{\partial}{\partial \dot{u}_{\alpha}}+\bar{U}_{\alpha i j} \frac{\partial}{\partial u_{\alpha, i j}}+\bar{U}_{\alpha i t} \frac{\partial}{\partial \dot{u}_{\alpha, i}}+\bar{U}_{\alpha t t} \frac{\partial}{\partial \ddot{u}_{\alpha}} \\
& \boldsymbol{b}=\bar{\Psi}_{\alpha i} \frac{\partial}{\partial \varphi_{\alpha, i}}+\bar{\Psi}_{\alpha t} \frac{\partial}{\partial \dot{\varphi}_{\alpha}}+\bar{\Psi}_{\alpha i j} \frac{\partial}{\partial \varphi_{\alpha, i j}}+\bar{\Psi}_{\alpha i t} \frac{\partial}{\partial \dot{\varphi}_{\alpha, i}}+\bar{\Psi}_{\alpha t t} \frac{\partial}{\partial \ddot{\varphi}_{\alpha}} \\
& \boldsymbol{c}=\bar{\Phi}_{\alpha \beta i} \frac{\partial}{\partial \phi_{\alpha \beta, i}}+\bar{\Phi}_{\alpha \beta t} \frac{\partial}{\partial \dot{\phi}_{\alpha \beta}}+\bar{\Phi}_{\alpha \beta i j} \frac{\partial}{\partial \phi_{\alpha \beta, i j}}+\bar{\Phi}_{\alpha \beta i t} \frac{\partial}{\partial \dot{\phi}_{\alpha \beta, i}}+\bar{\Phi}_{\alpha \beta t t} \frac{\partial}{\partial \ddot{\phi}_{\alpha \beta}}
\end{aligned}
$$

with

$$
\begin{aligned}
\bar{U}_{\alpha i} & =\mathrm{D}_{i}\left(U_{\alpha}-X_{k} u_{\alpha, k}-\tau \dot{u}_{\alpha}\right)+X_{k} u_{\alpha, k i}+\tau \dot{u}_{\alpha, i}, \\
\bar{U}_{\alpha t} & =\mathrm{D}_{t}\left(U_{\alpha}-X_{k} u_{\alpha, k}-\tau \dot{u}_{\alpha}\right)+X_{k} \dot{u}_{\alpha, k}+\tau \ddot{u}_{\alpha}, \\
\bar{U}_{\alpha i j} & =\mathrm{D}_{i} \mathrm{D}_{j}\left(U_{\alpha}-X_{k} u_{\alpha, k}-\tau \dot{u}_{\alpha}\right)+X_{k} u_{\alpha, k i j}+\tau \dot{u}_{\alpha, i j}, \\
\bar{U}_{\alpha i t} & =\mathrm{D}_{i} \mathrm{D}_{t}\left(U_{\alpha}-X_{k} u_{\alpha, k}-\tau \dot{u}_{\alpha}\right)+X_{k} \dot{u}_{\alpha, k i}+\tau \ddot{u}_{\alpha, i}, \\
\bar{U}_{\alpha t t} & =\mathrm{D}_{t} \mathrm{D}_{t}\left(U_{\alpha}-X_{k} u_{\alpha, k}-\tau \dot{u}_{\alpha}\right)+X_{k} \ddot{u}_{\alpha, k}+\tau \dddot{u}_{\alpha} .
\end{aligned}
$$

Similar expressions are given for the coefficients of the vectors $\boldsymbol{b}$ and $\boldsymbol{c}$ if in the above formulas the variables $U_{\alpha}$ and $u_{\alpha}$ are substituted by $\Psi_{\alpha}, \varphi_{\alpha}$ and $\Phi_{\alpha \beta}, \phi_{\alpha \beta}$, respectively.

The Lie-group $G$ is a group of invariance of the system $\Delta$ if and only if [16]

$$
\operatorname{pr}^{(2)} \boldsymbol{\nu}(\triangle)=0, \quad \text { whenever } \quad \triangle=0,
$$


for every infinitesimal generator $\boldsymbol{\nu}$. Applying the procedure (3.21) to the system of Eqs. (2.28)-(2.30), we get for the infinitesimal generator $\boldsymbol{\nu}$

$$
\begin{aligned}
& \rho\left(\bar{U}_{i t t}+\bar{\Psi}_{i t}\right)-\lambda\left(\bar{U}_{j j i}+\bar{\Phi}_{j j i}\right)-(\mu+\gamma)\left(\bar{U}_{i j j}+\bar{\Phi}_{i j j}\right)-\left.(\mu-\gamma)\left(\bar{U}_{j j i}+\bar{\Phi}_{j j i}\right)\right|_{\Delta=0}=0 \\
& d_{1}\left(\bar{\Psi}_{j j i}-\bar{\Phi}_{j j i t}\right)+\left(d_{2}+d_{3}\right)\left(\bar{\Psi}_{i j j}-\bar{\Phi}_{i j j t}\right)+\left(d_{2}-d_{3}\right)\left(\bar{\Psi}_{j j i}-\bar{\Phi}_{j i j t}\right)-\left.\rho\left(\bar{U}_{i t}+\bar{\Psi}_{i}\right)\right|_{\Delta=0}=0 \\
& d_{1} \delta_{i j}\left(\bar{\Psi}_{k k t}-\bar{\Phi}_{k k t t}\right)+\left(d_{2}+d_{3}\right)\left(\bar{\Psi}_{i j t}-\bar{\Phi}_{i j t t}\right)+\left(d_{2}-d_{3}\right)\left(\bar{\Psi}_{j i t}-\bar{\Phi}_{j i t t}\right) \\
& -c_{1}\left(\bar{\Phi}_{i k j k}-\bar{\Phi}_{i j k k}\right)-c_{2}\left(\bar{\Phi}_{j i k k}-\bar{\Phi}_{j k i k}+\bar{\Phi}_{k j i k}-\bar{\Phi}_{k i j k}\right)-c_{3}\left[\delta_{i j}\left(\bar{\Phi}_{l k l k}-\bar{\Phi}_{l l k k}\right)+\left(\bar{\Phi}_{l l j i}-\bar{\Phi}_{l j l i}\right)\right] \\
& -\lambda \delta_{i j}\left(\bar{U}_{l l}+\bar{\Phi}_{l l}\right)-(\mu+\gamma)\left(\bar{U}_{i j}+\bar{\Phi}_{i j}\right)-\left.(\mu-\gamma)\left(\bar{U}_{j i}+\bar{\Phi}_{j i}\right)\right|_{\Delta=0}=0 .
\end{aligned}
$$

From the condition above the form of the infinitesimal generator $\boldsymbol{\nu}$ is found

$$
\begin{aligned}
\tau & =d_{4} \\
X_{i} & =d_{i}+\epsilon_{i j k} a_{j} x_{k}, \\
U_{\alpha} & =c u_{\alpha}+\epsilon_{\alpha j k} a_{j} u_{k}-f_{\alpha}+\bar{u}_{\alpha}, \\
\Psi_{\alpha} & =c \varphi_{\alpha}+\epsilon_{\alpha j k} a_{j} \varphi_{k}+\dot{f}_{\alpha}+\bar{\varphi}_{\alpha}, \\
\Phi_{\alpha \beta} & =c \phi_{\alpha \beta}+\epsilon_{\alpha j k} a_{j} \phi_{k \beta}+\epsilon_{\beta j k} a_{j} \phi_{\alpha k}+f_{\alpha, \beta}+\bar{\phi}_{\alpha \beta},
\end{aligned}
$$

where $a_{j}, d_{i}$ and $c$ are arbitrary parameters. Moreover, $f_{\alpha}$ is a gauge function and $\bar{u}_{\alpha}, \bar{\varphi}_{\alpha}$, $\bar{\phi}_{\alpha \beta}$ are solutions of the Euler-Lagrange equations (2.20)-(2.22). The symmetry algebra is generated by the following vector fields:

$$
\begin{aligned}
& v^{1}=\frac{\partial}{\partial t} \\
& v_{i}^{2}=\frac{\partial}{\partial x_{i}} \\
& v_{i}^{3}=\epsilon_{i j k}\left(x_{j} \frac{\partial}{\partial x_{k}}+u_{j} \frac{\partial}{\partial u_{k}}+\varphi_{j} \frac{\partial}{\partial \varphi_{k}}+\phi_{l j} \frac{\partial}{\partial \phi_{l k}}+\phi_{j l} \frac{\partial}{\partial \phi_{k l}}\right) \quad \text { (rotations) } \\
& v^{4}=u_{i} \frac{\partial}{\partial u_{i}}+\varphi_{i} \frac{\partial}{\partial \varphi_{i}}+\phi_{i j} \frac{\partial}{\partial \phi_{i j}} \quad \text { (scaling) } \\
& v^{5}=-f_{i} \frac{\partial}{\partial u_{i}}+\dot{f}_{i} \frac{\partial}{\partial \varphi_{i}}+f_{i, j} \frac{\partial}{\partial \phi_{i j}} \quad \text { (gauge) } \\
& v^{6}=\bar{u}_{i} \frac{\partial}{\partial u_{i}}+\bar{\varphi}_{i} \frac{\partial}{\partial \varphi_{i}}+\bar{\phi}_{i j} \frac{\partial}{\partial \phi_{i j}} \quad \text { (addition of solutions). }
\end{aligned}
$$




\section{Conservation and balance laws}

Since the famous theorem of Noether [24], it is well-know that to each of the continuous symmetries of the Lagrangian a conservation law for the physical system corresponds.

The Lagrangian $\mathcal{L}$ depends on the first derivatives of the dependent field variables. In that case the infinitesimal criterion of invariance [16] says that a Lie group $G$ is a variational or divergence symmetry of $\mathcal{L}$ if and only if

$$
\operatorname{pr}^{(1)} \boldsymbol{\nu}(\mathcal{L})+\mathcal{L}\left(\mathrm{D}_{i} X_{i}+\mathrm{D}_{t} \tau\right)=\mathrm{D}_{i} B_{i}+\mathrm{D}_{t} B_{4}
$$

where $B_{i}$ and $B_{4}$ are opportune analytic functions. If $B_{i} \neq 0$ and $B_{4} \neq 0$, then $\boldsymbol{\nu}$ is the generator of a divergence symmetry of the Lagrangian $\mathcal{L}$. If $B_{4}=0$ and $B_{i}=0, \boldsymbol{\nu}$ generates a variational symmetry of $\mathcal{L}$. Every variational or divergence symmetry of the Lagrangian $\mathcal{L}$ is also a symmetry of the associated Euler-Lagrange equations. The inverse statement is not true.

After some standard calculations (see, e.g., Lazar [25]) we find the conservation law in characteristic form

$$
\mathrm{D}_{i} A_{i}+\mathrm{D}_{t} A_{4}+Q_{\alpha}^{u} E_{\alpha}^{u}(\mathcal{L})+Q_{\alpha}^{\varphi} E_{\alpha}^{\varphi}(\mathcal{L})+Q_{\alpha \beta}^{\phi} E_{\alpha \beta}^{\phi}(\mathcal{L})=0
$$

where the characteristics are defined by

$$
\begin{aligned}
Q_{\alpha}^{u} & =U_{\alpha}-X_{j} u_{\alpha, j}-\tau \dot{u}_{\alpha}, \\
Q_{\alpha}^{\varphi} & =\Psi_{\alpha}-X_{j} \varphi_{\alpha, j}-\tau \dot{\varphi}_{\alpha}, \\
Q_{\alpha \beta}^{\phi} & =\Phi_{\alpha \beta}-X_{j} \phi_{\alpha \beta, j}-\tau \dot{\phi}_{\alpha \beta} .
\end{aligned}
$$

Therefore, if the Euler-Lagrange equations (2.15)-(2.17) are fulfilled, we speak of a conservation law

$$
\mathrm{D}_{t} A_{4}+\mathrm{D}_{i} A_{i}=0
$$

where $A_{i}$ is the associated flux and $A_{4}$ is the conserved density. By the divergence theorem one finds the conservation law in integral form

$$
\int_{V} \mathrm{D}_{t} A_{4} \mathrm{~d} V+\int_{S} A_{i} n_{i} \mathrm{~d} S=0
$$

In case where the equality (4.6) is not fulfilled we speak of a balance law

$$
\mathrm{D}_{t} A_{4}+\mathrm{D}_{i} A_{i} \neq 0
$$

\subsection{Canonical currents}

If a variational or divergence symmetry is given, then the corresponding components $A_{4}$ and $A_{i}$ of the conservation law (4.6) are given by

$$
\begin{aligned}
& A_{4}=\mathcal{L} \tau+Q_{\alpha}^{u} \frac{\partial \mathcal{L}}{\partial \dot{u}_{\alpha}}+Q_{\alpha}^{\varphi} \frac{\partial \mathcal{L}}{\partial \dot{\varphi}_{\alpha}}+Q_{\alpha \beta}^{\phi} \frac{\partial \mathcal{L}}{\partial \dot{\phi}_{\alpha \beta}}-B_{4} \\
& A_{i}=\mathcal{L} X_{i}+Q_{\alpha}^{u} \frac{\partial \mathcal{L}}{\partial u_{\alpha, i}}+Q_{\alpha}^{\varphi} \frac{\partial \mathcal{L}}{\partial \varphi_{\alpha, i}}+Q_{\alpha \beta}^{\phi} \frac{\partial \mathcal{L}}{\partial \phi_{\alpha \beta, i}}-B_{i}
\end{aligned}
$$




\subsubsection{Translational invariance}

The translation acts only on the independent variables. The Lie-point group transformation of the translation in space and time is given by the formulas

$$
x_{i}^{\prime}=x_{i}+\varepsilon_{k} \delta_{k i}, \quad t^{\prime}=t+\varepsilon_{4} \delta_{44},
$$

leaving the field variables unchanged

$$
u_{\alpha}^{\prime}=u_{\alpha}, \quad \varphi_{\alpha}^{\prime}=\varphi_{\alpha}, \quad \phi_{\alpha \beta}^{\prime}=\phi_{\alpha \beta} .
$$

The components of the generator (3.11) corresponding to the infinitesimal transformations (4.11) and (4.12) take the form

$$
X_{k i}=\delta_{k i}, \quad \tau=\delta_{44}, \quad U_{\alpha}=0, \quad \Psi_{\alpha}=0, \quad \Phi_{\alpha \beta}=0 .
$$

Using Eqs. (4.9), (4.10) and (4.13), we obtain for the translational density and flux quantities

$$
\begin{aligned}
& A_{k i}=\mathcal{L} \delta_{k i}-u_{\alpha, k} \frac{\partial \mathcal{L}}{\partial u_{\alpha, i}}-\varphi_{\alpha, k} \frac{\partial \mathcal{L}}{\partial \varphi_{\alpha, i}}-\phi_{\alpha \beta, k} \frac{\partial \mathcal{L}}{\partial \phi_{\alpha \beta, i}}, \\
& A_{k 4}=-u_{\alpha, k} \frac{\partial \mathcal{L}}{\partial \dot{u}_{\alpha}}-\varphi_{\alpha, k} \frac{\partial \mathcal{L}}{\partial \dot{\varphi}_{\alpha}}-\phi_{\alpha \beta, k} \frac{\partial \mathcal{L}}{\partial \dot{\phi}_{\alpha \beta}}, \\
& A_{4 i}=-\dot{u}_{\alpha} \frac{\partial \mathcal{L}}{\partial u_{\alpha, i}}-\dot{\varphi}_{\alpha} \frac{\partial \mathcal{L}}{\partial \varphi_{\alpha, i}}-\dot{\phi}_{\alpha \beta} \frac{\partial \mathcal{L}}{\partial \phi_{\alpha \beta, i}} \\
& A_{44}=\mathcal{L}-\dot{u}_{\alpha} \frac{\partial \mathcal{L}}{\partial \dot{u}_{\alpha}}-\dot{\varphi}_{\alpha} \frac{\partial \mathcal{L}}{\partial \dot{\varphi}_{\alpha}}-\dot{\phi}_{\alpha \beta} \frac{\partial \mathcal{L}}{\partial \dot{\phi}_{\alpha \beta}} .
\end{aligned}
$$

In terms of the momentum $p_{i}$, the dislocation momentum flux $D_{i j}$, the force stress $\sigma_{i j}$ and pseudomoment stress $H_{i j k}$ they read as

$$
\begin{aligned}
P_{k i} & :=-A_{k i}=-\mathcal{L} \delta_{k i}-\sigma_{\alpha i} u_{\alpha, k}-D_{\alpha i} \varphi_{\alpha, k}+H_{\alpha \beta i} \phi_{\alpha \beta, k}, \\
\mathcal{P}_{k} & :=A_{k 4}=-p_{\alpha} u_{\alpha, k}-D_{\alpha \beta} \phi_{\alpha \beta, k}, \\
S_{i} & :=A_{4 i}=\sigma_{\alpha i} \dot{u}_{\alpha}+D_{\alpha i} \dot{\varphi}_{\alpha}-H_{\alpha \beta i} \dot{\phi}_{\alpha \beta}, \\
\mathcal{H} & :=-A_{44}=-\mathcal{L}+p_{\alpha} \dot{u}_{\alpha}+D_{\alpha \beta} \dot{\phi}_{\alpha \beta} .
\end{aligned}
$$

The tensor $P_{k i}$ is the canonical Eshelby stress tensor of dislocation gauge theory. The vector $\mathcal{P}_{k}$ is the canonical pseudomomentum or field momentum density and the vector $S_{i}$ is called the canonical field intensity or Poynting vector. The scalar $\mathcal{H}$ is the canonical energy density. They are related to each other by the following local conservation laws

$$
\begin{aligned}
\mathrm{D}_{t} \mathcal{P}_{k}-\mathrm{D}_{i} P_{k i} & =0 \\
\mathrm{D}_{t} \mathcal{H}-\mathrm{D}_{i} S_{i} & =0
\end{aligned}
$$

The first equation represents the canonical conservation law of pseudomomentum, while the second one constitutes the canonical conservation law of 
energy for the dislocation gauge theory. By the help of the Gauss theorem, these conservation laws appear in integral form

$$
\begin{aligned}
J_{k} & :=\int_{S} P_{k i} n_{i} \mathrm{~d} S-\int_{V} \mathrm{D}_{t} \mathcal{P}_{k} \mathrm{~d} V=0 \\
I & :=\int_{S} S_{i} n_{i} \mathrm{~d} S-\int_{V} \mathrm{D}_{t} \mathcal{H} \mathrm{d} V=0 .
\end{aligned}
$$

\subsubsection{Rotational invariance}

The three-dimensional rotations act on both, the independent and dependent variables. The infinitesimal transformations of the Lie group action are given by

$$
\begin{aligned}
x_{i}^{\prime} & =x_{i}+\epsilon_{i j k} \varepsilon_{j} x_{k}, \\
t^{\prime} & =t, \\
u_{\alpha}^{\prime} & =u_{\alpha}+\epsilon_{\alpha j k} \varepsilon_{j} u_{k}, \\
\varphi_{\alpha}^{\prime} & =\varphi_{\alpha}+\epsilon_{\alpha j k} \varepsilon_{j} \varphi_{k}, \\
\phi_{\alpha \beta}^{\prime} & =\phi_{\alpha \beta}+\left(\epsilon_{\alpha j k} \phi_{k \beta}+\epsilon_{\beta j k} \phi_{\alpha k}\right) \varepsilon_{j},
\end{aligned}
$$

from which the corresponding components of the generator (3.11) are calculated

$$
X_{i j}=\epsilon_{i j k} x_{k}, \quad U_{\alpha j}=\epsilon_{\alpha j k} u_{k}, \quad \Psi_{\alpha j}=\epsilon_{\alpha j k} \varphi_{k}, \quad \Phi_{\alpha \beta j}=\epsilon_{\alpha j k} \phi_{k \beta}+\epsilon_{\beta j k} \phi_{\alpha k} .
$$

Substituting these components into the Eqs. (4.9) and (4.10), we obtain

$$
\begin{gathered}
A_{k i}=\epsilon_{k m j}\left[x_{m}\left(\mathcal{L} \delta_{i j}-u_{\alpha, j} \frac{\partial \mathcal{L}}{\partial u_{\alpha, i}}-\varphi_{\alpha, j} \frac{\partial \mathcal{L}}{\partial \varphi_{\alpha, i}}-\phi_{\alpha \beta, j} \frac{\partial \mathcal{L}}{\partial \phi_{\alpha \beta, i}}\right)\right. \\
\left.+u_{m} \frac{\partial \mathcal{L}}{\partial u_{j, i}}+\varphi_{m} \frac{\partial \mathcal{L}}{\partial \varphi_{j, i}}+\phi_{m l} \frac{\partial \mathcal{L}}{\partial \phi_{j i, l}}+\phi_{l m} \frac{\partial \mathcal{L}}{\partial \phi_{l i, j}}\right], \\
A_{k 4}=\epsilon_{k m j}\left[u_{m} \frac{\partial \mathcal{L}}{\partial \dot{u}_{j}}+\varphi_{m} \frac{\partial \mathcal{L}}{\partial \dot{\varphi}_{j}}+\phi_{m l} \frac{\partial \mathcal{L}}{\partial \dot{\phi}_{j l}}+\phi_{l m} \frac{\partial \mathcal{L}}{\partial \dot{\phi}_{l j}}\right. \\
\left.-x_{j}\left(u_{\alpha, j} \frac{\partial \mathcal{L}}{\partial \dot{u}_{\alpha}}+\varphi_{\alpha, j} \frac{\partial \mathcal{L}}{\partial \dot{\varphi}_{\alpha}}+\phi_{\alpha \beta, j} \frac{\partial \mathcal{L}}{\partial \dot{\phi}_{\alpha \beta}}\right)\right] .
\end{gathered}
$$

We can introduce the total canonical angular-momentum tensor and the material inertia vector according

$$
\begin{aligned}
& M_{k i}:=-A_{k i}=\epsilon_{k m j}\left(x_{m} P_{j i}+\sigma_{j i} u_{m}+D_{j i} \varphi_{m}-H_{j l i} \phi_{m l}-H_{l j i} \phi_{l m}\right), \\
& \mathcal{M}_{k}:=A_{k 4}=\epsilon_{k m j}\left(x_{m} \mathcal{P}_{j}+p_{j} u_{m}+D_{j l} \phi_{m l}+D_{l j} \phi_{l m}\right) .
\end{aligned}
$$

It is possible to decompose the total canonical angular-momentum tensor and the material inertia vector into two parts

$$
M_{k i}=M_{k i}^{(\mathrm{o})}+M_{k i}^{(\mathrm{i})}, \quad \mathcal{M}_{k}=\mathcal{M}_{k}^{(\mathrm{o})}+\mathcal{M}_{k}^{(\mathrm{i})},
$$


where

$$
\begin{aligned}
M_{k i}^{(\mathrm{o})} & =\epsilon_{k m j} x_{m} P_{j i}, \\
M_{k i}^{(\mathrm{i})} & =\epsilon_{k m j}\left(\sigma_{j i} u_{m}+D_{j i} \varphi_{m}-H_{j l i} \phi_{m l}-H_{l j i} \phi_{l m}\right), \\
\mathcal{M}_{k}^{(\mathrm{o})} & =\epsilon_{k m j} x_{m} \mathcal{P}_{j}, \\
\mathcal{M}_{k}^{(\mathrm{i})} & =\epsilon_{k m j}\left(p_{j} u_{m}+D_{j l} \phi_{m l}+D_{l j} \phi_{l m}\right),
\end{aligned}
$$

are the orbital and intrinsic (or spin) angular-momentum tensors and material inertia vectors, respectively. For the balance of the material inertia vector we obtain

$$
\mathrm{D}_{t} \mathcal{M}_{k}-\mathrm{D}_{i} M_{k i}=\mathrm{D}_{t} \mathcal{M}_{k}^{(\mathrm{o})}-\mathrm{D}_{i} M_{k i}^{(\mathrm{o})}+\mathrm{D}_{t} \mathcal{M}_{k}^{(\mathrm{i})}-\mathrm{D}_{i} M_{k i}^{(\mathrm{i})}
$$

Due to the conservation of the pseudomomentum (4.22), the orbital parts become

$$
\mathrm{D}_{t} \mathcal{M}_{k}^{(\mathrm{o})}-\mathrm{D}_{i} M_{k i}^{(\mathrm{o})}=\epsilon_{k j m} P_{j m}
$$

Because of the rotational invariance, by using the minimal replacement (2.4) and the Euler-Lagrange equations (2.20)-(2.22) the balance law of the intrinsic angular-momentum becomes

$$
\begin{gathered}
\mathrm{D}_{t} \mathcal{M}_{k}^{(\mathrm{i})}-\mathrm{D}_{i} M_{k i}^{(\mathrm{i})}=\epsilon_{k m j}\left(v_{m} p_{j}-\sigma_{j l} \beta_{m l}+D_{j l} I_{m l}-\frac{1}{2} H_{j i l} T_{m i l}-H_{l i j} T_{l i m}\right. \\
\left.-\sigma_{l j} \phi_{l m}+D_{l j} \dot{\phi}_{l m}-H_{l i j} \phi_{l i, m}\right) .
\end{gathered}
$$

With Eqs. (4.18), (4.42) and (4.43) the rotational balance of the total angular momentum reads

$\mathrm{D}_{t} \mathcal{M}_{k}-\mathrm{D}_{i} M_{k i}=\epsilon_{k m j}\left(v_{m} p_{j}-\beta_{m l} \sigma_{j l}-\beta_{l m} \sigma_{l j}+I_{m l} D_{j l}+I_{l m} D_{l j}-\frac{1}{2} T_{m i l} H_{j i l}-T_{l i m} H_{l i j}\right)$.

Using the relations for the dislocation density tensor $T_{i k l}=\epsilon_{k l j} \alpha_{i j}$ and pseudomoment stress tensor $H_{i k l}=\epsilon_{k l j} H_{i j}$, we obtain

$$
\mathrm{D}_{t} \mathcal{M}_{k}-\mathrm{D}_{i} M_{k i}=\epsilon_{k m j}\left(v_{m} p_{j}-\beta_{m l} \sigma_{j l}-\beta_{l m} \sigma_{l j}+I_{m l} D_{j l}+I_{l m} D_{l j}-\alpha_{m l} H_{j l}-\alpha_{l m} H_{l j}\right) .
$$

In the case when the material is isotropic, by using the constitutive Eqs. (2.23)-(2.26), it can be shown that (4.44) becomes a conservation law. Using the Gauss theorem, we introduce the dynamical $\boldsymbol{L}$-integral of dislocation gauge theory:

$$
L_{k}:=\int_{S} M_{k i} n_{i} \mathrm{~d} S-\int_{V} \mathrm{D}_{t} \mathcal{M}_{k} \mathrm{~d} V
$$

\subsubsection{Scaling}

In the gauge field theory of dislocations the scaling group (dilatational group) is not a variational symmetry. The self-similarity is broken, since the defects like dislocations 
in the material introduce characteristic length scales. Still it is possible to calculate the broken scaling quantities. The scaling group acts in infinitesimal form on the independent and dependent variables in the following manner

$$
\begin{aligned}
x_{i}^{\prime} & =(1+\varepsilon) x_{i}, \\
t^{\prime} & =(1+\varepsilon) t, \\
u_{\alpha}^{\prime} & =\left(1+\varepsilon d_{u}\right) u_{\alpha}, \\
\varphi_{\alpha}^{\prime} & =\left(1+\varepsilon d_{\varphi}\right) \varphi_{\alpha}, \\
\phi_{\alpha \beta}^{\prime} & =\left(1+\varepsilon d_{\phi}\right) \phi_{\alpha \beta} .
\end{aligned}
$$

These transformation determines also the form for the components of the infinitesimal generator

$$
X_{i}=x_{i}, \quad \tau=t, \quad U_{\alpha}=d_{u} u_{\alpha}, \quad \Psi_{\alpha}=d_{\varphi} \varphi_{\alpha}, \quad \Phi_{\alpha \beta}=d_{\phi} \phi_{\alpha \beta},
$$

where $d_{u}, d_{\varphi}, d_{\phi}$ denote the (canonical) dimensions of the displacement vector $\boldsymbol{u}$ and the gauge potentials $\boldsymbol{\varphi}, \boldsymbol{\phi}$. If one substitutes the relation (4.52) into the Eqs. (4.9) and (4.10), one obtains for the scaling quantities

$$
\begin{aligned}
A_{i}=x_{i} \mathcal{L} & +\left(d_{u} u_{\alpha}-x_{k} u_{\alpha, k}-t \dot{u}_{\alpha}\right) \frac{\partial \mathcal{L}}{\partial u_{\alpha, i}}+\left(d_{\varphi} \varphi_{\alpha}-x_{k} \varphi_{\alpha, k}-t \dot{\varphi}_{\alpha}\right) \frac{\partial \mathcal{L}}{\partial \varphi_{\alpha, i}} \\
& +\left(d_{\phi} \phi_{\alpha \beta}-x_{k} \phi_{\alpha \beta, k}-t \dot{\phi}_{\alpha \beta}\right) \frac{\partial \mathcal{L}}{\partial \phi_{\alpha \beta, i}} \\
A_{4}=t & +\left(d_{u} u_{\alpha}-x_{k} u_{\alpha, k}-t \dot{u}_{\alpha}\right) \frac{\partial \mathcal{L}}{\partial \dot{u}_{\alpha}}+\left(d_{\varphi} \varphi_{\alpha}-x_{k} \varphi_{\alpha, k}-t \dot{\varphi}_{\alpha}\right) \frac{\partial \mathcal{L}}{\partial \dot{\varphi}_{\alpha}} \\
+ & \left(d_{\phi} \phi_{\alpha \beta}-x_{k} \phi_{\alpha \beta, k}-t \dot{\phi}_{\alpha \beta}\right) \frac{\partial \mathcal{L}}{\partial \dot{\phi}_{\alpha \beta}}
\end{aligned}
$$

In terms of force stress, pseudomoment stress and also with the canonical pseudo-momentum vector, the Poynting vector, the Eshelby stress tensor and the energy density the scaling flux densities become

$$
\begin{aligned}
Y_{i} & :=-A_{i}=x_{j} P_{j i}-t S_{i}+d_{u} \sigma_{j i} u_{j}+d_{\varphi} D_{j i} \varphi_{j}-d_{\phi} H_{j l i} \phi_{j l} \\
\mathcal{Y} & :=A_{4}=x_{j} \mathcal{P}_{j}-t \mathcal{H}+d_{u} p_{j} u_{j}+d_{\phi} D_{j l} \phi_{j l} .
\end{aligned}
$$

Eq. (4.55) is the dilatational vector flux and (4.56) is some kind of generalized action. The balance law of scaling symmetry reads

$$
\begin{aligned}
\mathrm{D}_{t} \mathcal{Y}-\mathrm{D}_{i} Y_{i}=( & \left.d_{u}+\frac{d-2}{2}\right)\left(p_{i} \dot{u}_{i}-u_{i, j} \sigma_{i j}\right)+\left(d_{\varphi}+\frac{d}{2}\right) p_{i} \varphi_{i}-\left(d_{\phi}+\frac{d}{2}\right) \sigma_{i j} \phi_{i j} \\
& +\left(d_{\phi}+\frac{d-2}{2}\right)\left(D_{i j} \dot{\phi}_{i j}-\frac{1}{2} H_{i j k} T_{i j k}\right)-\left(d_{\varphi}+\frac{d-2}{2}\right) D_{i j} \varphi_{i, j}
\end{aligned}
$$

Eq. (4.57) is the balance law for the scalar moment of momentum in the gauge theory of dislocations. It can be seen that we have two possibilities for the choice of $d_{\varphi}$ and $d_{\phi}$. The first choice is close to micropolar and micromorphic elasticity (see, 
e.g., Lubarda and Markenscoff [26], Lazar [25]). Then, the scaling dimensions of the field variables are determined according

$$
d_{u}=-\frac{d-2}{2}, \quad d_{\varphi}=-\frac{d}{2}, \quad d_{\phi}=-\frac{d}{2},
$$

where $d=n+1$ is the space-time dimension. In the present dynamic case for $d=4=3+1$, we obtain $d_{u}=-1, d_{\varphi}=-2$ and $d_{\phi}=-2$. With this choice (4.58), all the fields $\beta_{i j}, u_{i, j}$, $\phi_{i j}, v_{i}, \dot{u}_{i}$ and $\varphi_{i}$ have the same scaling dimension: $-\frac{d}{2}$. Thus, the minimal replacement (2.4) is scale invariant in the present case. Using (4.58), the balance law (4.57) reads

$$
\mathrm{D}_{t} \mathcal{Y}-\mathrm{D}_{i} Y_{i}=-2 \mathcal{L}_{\mathrm{di}}
$$

Thus, like in micromorphic elasticity the higher order terms break the scaling symmetry. In the present case $D_{i j}$ and $H_{i j k}$ break the scaling symmetry. The corresponding $M$ integral is given by

$$
M^{(1)}:=\int_{S} Y_{i} n_{i} \mathrm{~d} S-\int_{V} \mathrm{D}_{t} \mathcal{Y} \mathrm{d} V=2 \int_{V} \mathcal{L}_{\mathrm{di}} \mathrm{d} V
$$

The other choice for the scaling dimensions is

$$
d_{u}=-\frac{d-2}{2}, \quad d_{\varphi}=-\frac{d-2}{2}, \quad d_{\phi}=-\frac{d-2}{2} .
$$

It is obvious that this choice is like in Maxwell's field theory of electromagnetic fields, where scalar and vector fields have the same dimensions, namely $-\frac{d-2}{2}$ (see, e.g., Felsager [27]). It can be seen that now the minimal replacement (2.4) is not scaling invariant because $u_{i, j}$ has the dimension $-\frac{d}{2}$ and $\phi_{i j}$ has $-\frac{d-2}{2}$. With $d=4$, we obtain $d_{u}=-1$, $d_{\varphi}=-1$ and $d_{\phi}=-1$. Now the scaling balance law (4.57) reads

$$
\mathrm{D}_{t} \mathcal{Y}-\mathrm{D}_{i} Y_{i}=p_{i} \varphi_{i}-\sigma_{i j} \phi_{i j}
$$

It is obvious that the source terms of the Euler-Lagrange equations (2.16) and (2.17) break the scaling symmetry. The corresponding $M$-integral is now given by

$$
M^{(2)}:=\int_{S} Y_{i} n_{i} \mathrm{~d} S-\int_{V} \mathrm{D}_{t} \mathcal{Y} \mathrm{d} V=-\int_{V}\left(p_{i} \varphi_{i}-\sigma_{i j} \phi_{i j}\right) \mathrm{d} V .
$$

\subsubsection{Gauge symmetry}

The gauge symmetry acts in the following way

$$
x_{i}^{\prime}=x_{i}, \quad t^{\prime}=t, \quad u_{\alpha}^{\prime}=u_{\alpha}-\varepsilon f_{\alpha}, \quad \varphi_{\alpha}^{\prime}=\varphi_{\alpha}+\varepsilon \dot{f}_{\alpha}, \quad \phi_{\alpha \beta}^{\prime}=\phi_{\alpha \beta}+\varepsilon f_{\alpha, \beta} .
$$

Then the components of the generator (3.11) take the form

$$
X_{k i}=0, \quad \tau=0, \quad U_{\alpha}=-f_{\alpha}, \quad \Psi_{\alpha}=\dot{f}_{\alpha}, \quad \Phi_{\alpha \beta}=f_{\alpha, \beta} .
$$


The density and flux current which can be derived from the infinite dimensional group generator (3.34) by using the formulas (4.9) and (4.10) have the following form

$$
\begin{aligned}
G_{i} & :=-A_{i}=-\sigma_{j i} f_{j}+D_{j i} \dot{f}_{j}-H_{j k i} f_{j, k} \\
\mathcal{G} & :=A_{4}=-p_{j} f_{j}+D_{j i} f_{j, i} .
\end{aligned}
$$

The corresponding conservation law reads

$$
\mathrm{D}_{t} \mathcal{G}-\mathrm{D}_{i} G_{i}=f_{j, k i} H_{j[k i]}=0
$$

The global conservation law is

$$
\int_{S} G_{i} n_{i} \mathrm{~d} S-\int_{V} \mathrm{D}_{t} \mathcal{G} \mathrm{d} V=0
$$

\subsubsection{Addition of solutions}

The vector field $v^{6}$ is a generator of a divergence symmetry. The addition of solutions is given by

$$
x_{i}^{\prime}=x_{i}, \quad t^{\prime}=t, \quad u_{\alpha}^{\prime}=u_{\alpha}+\varepsilon \bar{u}_{\alpha}^{\prime}, \quad \varphi_{\alpha}^{\prime}=\varphi_{\alpha}+\varepsilon \bar{\varphi}_{\alpha}, \quad \phi_{\alpha \beta}^{\prime}=\phi_{\alpha \beta}+\varepsilon \bar{\phi}_{\alpha \beta} .
$$

Then the components of the generator (3.11) take the form

$$
X_{k i}=0, \quad \tau=0, \quad U_{\alpha}=\bar{u}_{\alpha}, \quad \Psi_{\alpha}=\bar{\varphi}_{\alpha}, \quad \Phi_{\alpha \beta}=\bar{\phi}_{\alpha \beta} .
$$

Using Betti's reciprocal theorem, the fields $B_{i}$ and $B_{4}$ are of the form

$$
\begin{aligned}
& B_{i}=-u_{\alpha} \bar{\sigma}_{\alpha i}-\varphi_{\alpha} \bar{D}_{\alpha i}+\phi_{\alpha \beta} \bar{H}_{\alpha \beta i}, \\
& B_{4}=u_{\alpha} \bar{p}_{\alpha}+\phi_{\alpha \beta} \bar{D}_{\alpha \beta} .
\end{aligned}
$$

The notation $\bar{\sigma}_{\alpha i}, \bar{D}_{\alpha i}$ and $\bar{H}_{\alpha \beta i}$ means that $\boldsymbol{u}, \boldsymbol{\varphi}$ and $\boldsymbol{\phi}$ are replaced by $\overline{\boldsymbol{u}}, \overline{\boldsymbol{\varphi}}$ and $\overline{\boldsymbol{\phi}}$, respectively. Finally, the conserved fluxes are of the form

$$
\begin{aligned}
& A_{i}=-\bar{u}_{\alpha} \sigma_{\alpha i}-\bar{\varphi}_{\alpha} D_{\alpha i}+\bar{\phi}_{\alpha \beta} H_{\alpha \beta i}+u_{\alpha} \bar{\sigma}_{\alpha i}+\varphi_{\alpha} \bar{D}_{\alpha i}-\phi_{\alpha \beta} \bar{H}_{\alpha \beta i}, \\
& A_{4}=\bar{u}_{\alpha} p_{\alpha}+\bar{\phi}_{\alpha \beta} D_{\alpha \beta}-u_{\alpha} \bar{p}_{\alpha}-\phi_{\alpha \beta} \bar{D}_{\alpha \beta} .
\end{aligned}
$$

The corresponding conservation law is a manifestation of Betti's reciprocal theorem for the gauge theory of dislocations and is a consequence of the linearity of $\Delta=0 \quad[28,29]$. In integral form the dynamical Betti reciprocal theorem is given by

$$
\begin{gathered}
\int_{S}\left(u_{\alpha} \bar{\sigma}_{\alpha i}+\varphi_{\alpha} \bar{D}_{\alpha i}-\phi_{\alpha \beta} \bar{H}_{\alpha \beta i}\right) n_{i} \mathrm{~d} S-\int_{V} \mathrm{D}_{t}\left(u_{\alpha} \bar{p}_{\alpha}+\phi_{\alpha \beta} \bar{D}_{\alpha \beta}\right) \mathrm{d} V= \\
\int_{S}\left(\bar{u}_{\alpha} \sigma_{\alpha i}+\bar{\varphi}_{\alpha} D_{\alpha i}-\bar{\phi}_{\alpha \beta} H_{\alpha \beta i}\right) n_{i} \mathrm{~d} S-\int_{V} \mathrm{D}_{t}\left(\bar{u}_{\alpha} p_{\alpha}+\bar{\phi}_{\alpha \beta} D_{\alpha \beta}\right) \mathrm{d} V .
\end{gathered}
$$




\subsection{Gauge-invariant currents}

Although all local conservation and balance laws for the total system can be brought in the gauge invariant form, this is not true for all the currents. Only the canonical currents (4.18)-(4.21) coming from the translational symmetry can be written in terms of gauge invariant quantities. For rewriting $P_{k i}$, we use the Eq. (2.4) for the distortion, the dislocation current and torsion density tensor (2.8) and the Eq. (2.22). For $\mathcal{P}_{k}$ we use also the same equations as for the canonical Eshelby-stress tensor, but instead of Eq. (2.22), Eq. (2.21) is now needed. For rewriting $\mathcal{H}$, we use the Eq. (2.4) for the kinematical velocity, the dislocation current density (2.8) and also the Eq. (2.21). For $S_{i}$ we use the same equations as for the canonical energy density, but instead of (2.21), Eq. (2.22) is used. The result reads as

$$
\begin{aligned}
P_{k i} & =P_{k i}^{(\mathrm{g})}-\mathrm{D}_{t}\left(D_{\alpha i} \phi_{\alpha k}\right)+\mathrm{D}_{\beta}\left(H_{\alpha \beta i} \phi_{\alpha k}\right), \\
\mathcal{P}_{k} & =\mathcal{P}_{k}^{(\mathrm{g})}-\mathrm{D}_{\beta}\left(D_{\alpha \beta} \phi_{\alpha k}\right), \\
S_{i} & =S_{i}^{(\mathrm{g})}+\mathrm{D}_{t}\left(D_{\alpha i} \varphi_{\alpha}\right)-\mathrm{D}_{\beta}\left(H_{\alpha \beta i} \varphi_{\alpha}\right), \\
\mathcal{H} & =\mathcal{H}^{(\mathrm{g})}+\mathrm{D}_{\beta}\left(D_{\alpha \beta} \varphi_{\alpha}\right),
\end{aligned}
$$

where the gauge-invariant currents are defined by

$$
\begin{aligned}
P_{k i}^{(\mathrm{g})} & :=-\mathcal{L} \delta_{k i}-\sigma_{\alpha i} \beta_{\alpha k}+D_{\alpha i} I_{\alpha k}-H_{\alpha \beta i} T_{\alpha \beta k}, \\
\mathcal{P}_{k}^{(\mathrm{g})} & :=-p_{\alpha} \beta_{\alpha k}+D_{\alpha \beta} T_{\alpha \beta k} \\
S_{i}^{(\mathrm{g})} & :=\sigma_{\alpha i} v_{\alpha}-H_{\alpha \beta i} I_{\alpha \beta} \\
\mathcal{H}^{(\mathrm{g})} & :=-\mathcal{L}+p_{\alpha} v_{\alpha}+D_{\alpha \beta} I_{\alpha \beta} .
\end{aligned}
$$

From the canonical local conservation laws (4.18)-(4.21) we can obtain the gauge invariant conservation laws

$$
\begin{aligned}
& \mathrm{D}_{t} \mathcal{P}_{k}^{(\mathrm{g})}-\mathrm{D}_{i} P_{k i}^{(\mathrm{g})}=0 \\
& \mathrm{D}_{t} \mathcal{H}^{(\mathrm{g})}-\mathrm{D}_{i} S_{i}^{(\mathrm{g})}=0
\end{aligned}
$$

since the divergence terms cancel each other out and

$$
\mathrm{D}_{i} \mathrm{D}_{\beta}\left(H_{\alpha \beta i} \phi_{\alpha k}\right)=0, \quad \mathrm{D}_{i} \mathrm{D}_{\beta}\left(H_{\alpha \beta i} \varphi_{\alpha}\right)=0
$$

because of the antisymmetry of $H_{i[j k]}$ in the last two indices. By the help of the Gauss theorem, we get the momentum and energy conservation laws for the gauge invariant quantities in integral form

$$
\begin{aligned}
J_{k}^{(\mathrm{g})} & :=\int_{S} P_{k i}^{(\mathrm{g})} n_{i} \mathrm{~d} S-\int_{V} \mathrm{D}_{t} \mathcal{P}_{k}^{(\mathrm{g})} \mathrm{d} V=0, \\
I^{(\mathrm{g})} & :=\int_{S} S_{i}^{(\mathrm{g})} n_{i} \mathrm{~d} S-\int_{V} \mathrm{D}_{t} \mathcal{H}^{(\mathrm{g})} \mathrm{d} V=0 .
\end{aligned}
$$




\section{Configurational force, moment and power}

In this section we decompose the total Lagrangian into its elastic and dislocation parts. Both parts are coupled by the Euler-Lagrange equations. These coupling terms will appear as additional configurational forces, vector and scalar momenta. Consequently, the total system is conserved but they are not conserved separately. There is an exchange between the elastic and dislocation subsystems.

\subsection{Translations in space and time}

In the last section the conservation laws for the canonical pseudomomentum, material inertia vector and energy density have been calculated. These conservation laws exist only if the total Lagrangian $\mathcal{L}$ composed of the kinetic and potential energies of the elastic and the dislocation field part is taken into account. If we consider only one part of the field, the canonical quantities are not any more conserved and as a consequence configurational forces, moments and power appear. We give the currents for both parts of the total system, the elastic part $\mathcal{L}_{\mathrm{e}}$ and the dislocation part $\mathcal{L}_{\mathrm{di}}$ of the total Lagrangian density.

\subsubsection{Elastic part}

For the elastic part $\mathcal{L}_{\mathrm{e}}$ we obtain from (4.9)-(4.10) for the Eqs. (4.18)-(4.21) the following canonical quantities of the elastic subsystem

$$
\begin{aligned}
P_{k i}^{(\mathrm{e})} & :=-\mathcal{L}_{\mathrm{e}} \delta_{k i}-\sigma_{\alpha i} u_{\alpha, k}, \\
\mathcal{P}_{k}^{(\mathrm{e})} & :=-p_{\alpha} u_{\alpha, k} \\
S_{i}^{(\mathrm{e})} & :=\sigma_{\alpha i} \dot{u}_{\alpha} \\
\mathcal{H}^{(\mathrm{e})} & :=-\mathcal{L}_{\mathrm{e}}+p_{\alpha} \dot{u}_{\alpha} .
\end{aligned}
$$

The local conservation laws (4.22) and (4.23) become now balance laws. At the right hand sides appear source terms called canonical configurational force and power. From Eqs. (5.1)-(5.4) we get

$$
\begin{aligned}
& \mathrm{D}_{t} \mathcal{P}_{k}^{(\mathrm{e})}-\mathrm{D}_{i} P_{k i}^{(\mathrm{e})}=\mathcal{F}_{k}, \\
& \mathrm{D}_{t} \mathcal{H}^{(\mathrm{e})}-\mathrm{D}_{i} S_{i}^{(\mathrm{e})}=\mathcal{W},
\end{aligned}
$$

where the configurational force and power densities read

$$
\begin{aligned}
& \mathcal{F}_{k}=p_{\alpha} \varphi_{\alpha, k}-\sigma_{\alpha \beta} \phi_{\alpha \beta, k}, \\
& \mathcal{W}=-p_{\alpha} \dot{\varphi}_{\alpha}+\sigma_{\alpha \beta} \dot{\phi}_{\alpha \beta} .
\end{aligned}
$$

The corresponding balance laws in integral form are given by

$$
\begin{aligned}
J_{k}^{(\mathrm{e})} & :=\int_{S} P_{k i}^{(\mathrm{e})} n_{i} \mathrm{~d} S-\int_{V} \mathrm{D}_{t} \mathcal{P}_{k}^{(\mathrm{e})} \mathrm{d} V=-\int_{V} \mathcal{F}_{k} \mathrm{~d} V, \\
I^{(\mathrm{e})} & :=\int_{S} S_{i}^{(\mathrm{e})} n_{i} \mathrm{~d} S-\int_{V} \mathrm{D}_{t} \mathcal{H}^{(\mathrm{e})} \mathrm{d} V=-\int_{V} \mathcal{W} \mathrm{d} V .
\end{aligned}
$$


A similar expression like (5.7) was earlier given by Kadić and Edelen [12]. They called this force the 'elastic excess' force.

For the elastic part $\mathcal{L}_{\mathrm{e}}$ of the total Lagrangian $\mathcal{L}$ the gauge invariant currents $(4.81)-$ (4.84) are

$$
\begin{aligned}
P_{k i}^{(\mathrm{e}-\mathrm{g})} & :=-\mathcal{L}_{\mathrm{e}} \delta_{k i}-\sigma_{\alpha i} \beta_{\alpha k}, \\
\mathcal{P}_{k}^{(\mathrm{e}-\mathrm{g})} & :=-p_{\alpha} \beta_{\alpha k}, \\
S_{i}^{(\mathrm{e}-\mathrm{g})} & :=\sigma_{\alpha i} v_{\alpha}, \\
\mathcal{H}^{(\mathrm{e}-\mathrm{g})} & :=-\mathcal{L}_{\mathrm{e}}+p_{\alpha} v_{\alpha} .
\end{aligned}
$$

From Eqs. (5.11)-(5.14), the balance laws can be written in the following form

$$
\begin{aligned}
& \mathrm{D}_{t} \mathcal{P}_{k}^{(\mathrm{e}-\mathrm{g})}-\mathrm{D}_{i} P_{k i}^{(\mathrm{e}-\mathrm{g})}=\mathcal{F}_{k}^{(\mathrm{g})}, \\
& \mathrm{D}_{t} \mathcal{H}^{(\mathrm{e}-\mathrm{g})}-\mathrm{D}_{i} S_{i}^{(\mathrm{e}-\mathrm{g})}=\mathcal{W}^{(\mathrm{g})},
\end{aligned}
$$

where

$$
\begin{aligned}
\mathcal{F}_{k}^{(\mathrm{g})} & =-p_{i} I_{i k}+\sigma_{i j} T_{i j k}, \\
\mathcal{W}^{(\mathrm{g})} & =\sigma_{i j} I_{i j},
\end{aligned}
$$

are the gauge invariant configurational force and power densities. In Eq. (5.17) we have two contributions to the configurational force density. The first term is the force density caused by a dislocation current density $I_{i k}$ in presence of a momentum field $p_{i}$ (see also $[30,31,32])$. The second term is the Peach-Koehler force density [15] recovered in the gauge theory of dislocations. It gives the force density caused by a dislocation density $T_{i j k}$ in presence of a stress field $\sigma_{i j}$. Here we call (5.17) the dynamical Peach-Koehler force density. Eq. (5.18) is the power density lost from a moving dislocation to the medium $[33,30,32]$. If we multiply $(2.20)$ by $v_{i}$ and the second equation of $(2.9)$ by $\sigma_{i j}$ and add each other, we get

$$
v_{i} \dot{p}_{i}+\sigma_{i j} \dot{\beta}_{i j}-\mathrm{D}_{j}\left(v_{i} \sigma_{i j}\right)=\sigma_{i j} I_{i j}
$$

Therefore, (5.18) is the rating density of the elastic material. The corresponding $\boldsymbol{J}$ and I-integrals read

$$
\begin{aligned}
J_{k}^{(\mathrm{e}-\mathrm{g})} & :=\int_{S} P_{k i}^{(\mathrm{e}-\mathrm{g})} n_{i} \mathrm{~d} S-\int_{V} \mathrm{D}_{t} \mathcal{P}_{k}^{(\mathrm{e}-\mathrm{g})} \mathrm{d} V=-\int_{V} \mathcal{F}_{k}^{(\mathrm{g})} \mathrm{d} V \\
I^{(\mathrm{e}-\mathrm{g})} & :=\int_{S} S_{i}^{(\mathrm{e}-\mathrm{g})} n_{i} \mathrm{~d} S-\int_{V} \mathrm{D}_{t} \mathcal{H}^{(\mathrm{e}-\mathrm{g})} \mathrm{d} V=-\int_{V} \mathcal{W}^{(\mathrm{g})} \mathrm{d} V .
\end{aligned}
$$

\section{Static case}

For a static theory the Eshelby stress tensors of the elastic subsystem have the following form

$$
\begin{aligned}
P_{k i}^{(\mathrm{e})} & =W_{\mathrm{e}} \delta_{k i}-\sigma_{\alpha i} u_{\alpha, k}, \\
P_{k i}^{(\mathrm{e}-\mathrm{g})} & =W_{\mathrm{e}} \delta_{k i}-\sigma_{\alpha i} \beta_{\alpha k},
\end{aligned}
$$


with the associated $\boldsymbol{J}$-integrals

$$
\begin{aligned}
J_{k}^{(\mathrm{e})} & =\int_{S} P_{k i}^{(\mathrm{e})} n_{i} \mathrm{~d} S=\int_{V} \mathcal{F}^{(\mathrm{Sta})} \mathrm{d} V=\int_{V} \sigma_{i j} \phi_{i j, k} \mathrm{~d} V, \\
J_{k}^{(\mathrm{e}-\mathrm{g})} & =\int_{S} P_{k i}^{(\mathrm{e}-\mathrm{g})} n_{i} \mathrm{~d} S=\int_{V} \mathcal{F}^{(\mathrm{PK})} \mathrm{d} V=-\int_{V} \sigma_{i j} T_{i j k} \mathrm{~d} V=\int_{V} \epsilon_{k j l} \sigma_{i j} \alpha_{i l} \mathrm{~d} V,
\end{aligned}
$$

where $T_{i j k}=\epsilon_{l j k} \alpha_{i l}$ with the usual dislocation density tensor $\alpha_{i l}$. The gauge invariant Eshelby stress tensor (5.23) is in agreement with the Eshelby stress tensor in incompatible elasticity derived by Kirchner [34], Lazar and Kirchner [35]. It can be seen that the configurational force density in (5.24) is just given in terms of the force stress tensor and the gradient of the plastic distortion. But this is not the correct Peach-Koehler force. On the other hand, the gauge invariant expression (5.25) gives the correct gauge-invariant Peach-Koehler force.

\subsubsection{Dislocation part}

In the same manner we obtain for the canonical currents of the dislocation part

$$
\begin{aligned}
P_{k i}^{(\mathrm{d})} & :=-\mathcal{L}_{\mathrm{di}} \delta_{k i}-D_{\alpha i} \varphi_{\alpha, k}+H_{\alpha \beta i} \phi_{\alpha \beta, k}, \\
\mathcal{P}_{k}^{(\mathrm{d})} & :=-D_{\alpha \beta} \phi_{\alpha \beta, k}, \\
S_{i}^{(\mathrm{d})} & :=D_{\alpha i} \dot{\varphi}_{\alpha}-H_{\alpha \beta i} \dot{\phi}_{\alpha \beta}, \\
\mathcal{H}^{(\mathrm{d})} & :=-\mathcal{L}_{\mathrm{di}}+D_{\alpha \beta} \dot{\phi}_{\alpha \beta}
\end{aligned}
$$

with the $\boldsymbol{J}$ and $I$-integrals of the dislocation subsystem

$$
\begin{aligned}
J_{k}^{(\mathrm{d})} & :=\int_{S} P_{k i}^{(\mathrm{d})} n_{i} \mathrm{~d} S-\int_{V} \mathrm{D}_{t} \mathcal{P}_{k}^{(\mathrm{d})} \mathrm{d} V=\int_{V} \mathcal{F}_{k} \mathrm{~d} V, \\
I^{(\mathrm{d})} & :=\int_{S} S_{i}^{(\mathrm{d})} n_{i} \mathrm{~d} S-\int_{V} \mathrm{D}_{t} \mathcal{H}^{(\mathrm{d})} \mathrm{d} V=\int_{V} \mathcal{W} \mathrm{d} V .
\end{aligned}
$$

The gauge invariant currents of the dislocation part $\mathcal{L}_{\text {di }}$ of the total Lagrangian $\mathcal{L}$ read

$$
\begin{aligned}
P_{k i}^{(\mathrm{d}-\mathrm{g})} & :=-\mathcal{L}_{\mathrm{di}} \delta_{k i}+D_{\alpha i} I_{\alpha k}-H_{\alpha \beta i} T_{\alpha \beta k}, \\
\mathcal{P}_{k}^{(\mathrm{d}-\mathrm{g})} & :=D_{\alpha \beta} T_{\alpha \beta k}, \\
S_{i}^{(\mathrm{d}-\mathrm{g})} & :=-H_{\alpha \beta i} I_{\alpha \beta}, \\
\mathcal{H}^{(\mathrm{d}-\mathrm{g})} & :=-\mathcal{L}_{\mathrm{di}}+D_{\alpha \beta} I_{\alpha \beta} .
\end{aligned}
$$

From them the configurational force and power can be calculated in the same way, as we did for the elastic part. If we use the Eqs. (5.26)-(5.29) and (5.32)-(5.35) for the calculation of the canonical and gauge invariant force and power, the same results as in the Eqs. (5.15), (5.16) and (5.17), (5.18) appear, but with opposite signs. The result is

$$
\begin{aligned}
J_{k}^{(\mathrm{d}-\mathrm{g})} & :=\int_{S} P_{k i}^{(\mathrm{d}-\mathrm{g})} n_{i} \mathrm{~d} S-\int_{V} \mathrm{D}_{t} \mathcal{P}_{k}^{(\mathrm{d}-\mathrm{g})} \mathrm{d} V=\int_{V} \mathcal{F}_{k}^{(\mathrm{g})} \mathrm{d} V, \\
I^{(\mathrm{d}-\mathrm{g})} & :=\int_{S} S_{i}^{(\mathrm{d}-\mathrm{g})} n_{i} \mathrm{~d} S-\int_{V} \mathrm{D}_{t} \mathcal{H}^{(\mathrm{d}-\mathrm{g})} \mathrm{d} V=\int_{V} \mathcal{W}^{(\mathrm{g})} \mathrm{d} V .
\end{aligned}
$$


If we multiply (2.22) by $I_{i j}$ and the second equation of (2.10) by $H_{i j k}$ and add each other, we obtain

$$
I_{i j} \dot{D}_{i j}+H_{i j k} \dot{T}_{i j k}+\mathrm{D}_{k}\left(I_{i j} H_{i j k}\right)=-\sigma_{i j} I_{i j}
$$

This is the power density lost by moving dislocations.

Finally, the $\boldsymbol{J}$ and the $I$ integrals of the total system may be decomposed into its elastic and dislocation parts according to

$$
\begin{gathered}
J_{k}=J_{k}^{(\mathrm{e})}+J_{k}^{(\mathrm{d})}=0, \\
I=I^{(\mathrm{e})}+I^{(\mathrm{d})}=0 .
\end{gathered}
$$

The configurational force and power densities appearing in the elastic and dislocation subsystems are the result of the interaction of both subsystems. Without interaction between the elastic and dislocation parts no configurational force and power densities would appear and $\boldsymbol{J}^{(\mathrm{e})}=0, I^{(\mathrm{e})}=0 \boldsymbol{J}^{(\mathrm{d})}=0, I^{(\mathrm{d})}=0$. Of course the same is true for the gauge invariance parts.

\section{Static case}

The static Eshelby stress tensors of the dislocation subsystem are

$$
\begin{aligned}
P_{k i}^{(\mathrm{d})} & =W_{\mathrm{di}} \delta_{k i}+H_{\alpha \beta i} \phi_{\alpha \beta, k}=W_{\mathrm{di}} \delta_{k i}-\epsilon_{i \beta l} H_{\alpha l} \phi_{\alpha \beta, k}, \\
P_{k i}^{(\mathrm{d}-\mathrm{g})} & =W_{\mathrm{di}} \delta_{k i}-H_{\alpha \beta i} T_{\alpha \beta k}=W_{\mathrm{di}} \delta_{k i}+\epsilon_{i \beta l} H_{\alpha l} T_{\alpha \beta k},
\end{aligned}
$$

with the $\boldsymbol{J}$ integrals

$$
\begin{aligned}
J_{k}^{(\mathrm{d})} & =\int_{S} P_{k i}^{(\mathrm{d})} n_{i} \mathrm{~d} S=-\int_{V} \mathcal{F}^{(\mathrm{Sta})} \mathrm{d} V=-\int_{V} \sigma_{i j} \phi_{i j, k} \mathrm{~d} V, \\
J_{k}^{(\mathrm{d}-\mathrm{g})} & =\int_{S} P_{k i}^{(\mathrm{d}-\mathrm{g})} n_{i} \mathrm{~d} S=-\int_{V} \mathcal{F}^{(\mathrm{PK})} \mathrm{d} V=\int_{V} \sigma_{i j} T_{i j k} \mathrm{~d} V .
\end{aligned}
$$

For a dislocation theory without force stresses, $\sigma_{i j}=0$, the Eshelby stress tensors (5.41) and (5.42) are divergenceless and the $\boldsymbol{J}$ integrals (5.43) and (5.44) are zero. This is the case for a (force) stress-free configuration with pseudo-moment stresses only. Then the Euler-Lagrange equation (2.22) takes the form

$$
\mathrm{D}_{i} H_{\alpha \beta i}=0 .
$$

If we use the so-called Einstein choice $c_{2}=-c_{1}$ and $c_{3}=-2 c_{1}[36,37]$, Eq. (5.45) (or Eq. (2.30)) reduces to the incompatibility condition for the elastic strain [22]: inc $\boldsymbol{\epsilon}=0$, where $\boldsymbol{\epsilon}=\frac{1}{2}\left(\boldsymbol{\beta}+\boldsymbol{\beta}^{\mathrm{T}}\right)$. Anyway, the Eshelby stress tensors (5.41) and (5.42) are the Eshelby stress tensors of the dislocation part. Recently, Li et al. [38] and Li [39] have used such an Eshelby stress tensor of dislocations without interaction with a force stress tensor. They called it compatibility momentum tensor. Furthermore, they have found conservation laws associated with compatibility conditions of continua. But, in fact, they investigated the static dislocation part without force stresses and they found (5.45) as compatibility condition and the $\boldsymbol{J}^{(\mathrm{d})}, \boldsymbol{L}^{(\mathrm{d})}$ and $M^{(\mathrm{d})}$ integrals of the dislocation subsystem. It is obvious that such conservation laws are not a "new" class of conservation laws. If the PeachKoehler force is zero, they are conservation laws. Thus, it is misleading to call (5.41) a compatibility momentum tensor. 


\subsection{Rotations in space}

\subsubsection{Elastic part}

In the same way as we did for the translations, we find for the canonical currents for the elastic part of the system described by $\mathcal{L}_{\text {el }}$

$$
\begin{aligned}
& M_{k i}^{(\mathrm{e})}:=\epsilon_{k m j}\left(x_{m} P_{j i}^{(\mathrm{e})}+u_{m} \sigma_{j i}\right), \\
& \mathcal{M}_{k}^{(\mathrm{e})}:=\epsilon_{k m j}\left(x_{m} \mathcal{P}_{j}^{(\mathrm{e})}+u_{m} p_{j}\right),
\end{aligned}
$$

where $P_{j i}^{(\mathrm{e})}$ and $\mathcal{P}_{j}^{(\mathrm{e})}$ are given by (5.1) and (5.2), respectively. The rotational balance law of the elastic part is calculated

$$
\mathrm{D}_{t} \mathcal{M}_{k}^{(\mathrm{e})}-\mathrm{D}_{i} M_{k i}^{(\mathrm{e})}=\epsilon_{k m j}\left(x_{m} \mathcal{F}_{j}+\left(v_{m} p_{j}-\beta_{m l} \sigma_{j l}-\beta_{l m} \sigma_{l j}\right)-\varphi_{m} p_{j}+\phi_{m l} \sigma_{j l}+\phi_{l m} \sigma_{l j}\right),
$$

where so-called configurational vector moments appear as source terms. In Eq. (5.48) the first part is a moment produced by the configurational force $\mathcal{F}_{j}$, the next three parts are the isotropy condition which vanishes if the constitutive relation between $\boldsymbol{\sigma}$ and $\boldsymbol{\beta}$ is isotropic. The other source terms are caused by the gauge potential which are the plastic fields. The $\boldsymbol{L}$ integral reads now as

$$
\begin{gathered}
L_{k}^{(\mathrm{e})}:=\int_{S} M_{k i}^{(\mathrm{e})} n_{i} \mathrm{~d} S-\int_{V} \mathrm{D}_{t} \mathcal{M}_{k}^{(\mathrm{e})} \mathrm{d} V=\int_{V} \epsilon_{k j m}\left(x_{m} \mathcal{F}_{j}+\left(v_{m} p_{j}-\beta_{m l} \sigma_{j l}-\beta_{l m} \sigma_{l j}\right)\right. \\
\left.-\varphi_{m} p_{j}+\phi_{m l} \sigma_{j l}+\phi_{l m} \sigma_{l j}\right) \mathrm{d} V .
\end{gathered}
$$

\section{Static case}

In the static case, the angular momentum tensor (5.46) is given in terms of the static Eshelby stress tensor (5.22). Eventually, the static $\boldsymbol{L}$-integral of the elastic subsystem reads

$$
L_{k}^{(\mathrm{e})}=\int_{S} M_{k i}^{(\mathrm{e})} n_{i} \mathrm{~d} S=\int_{V} \epsilon_{k j m}\left(x_{m} \mathcal{F}_{j}^{(\mathrm{Sta})}-\left(\beta_{m l} \sigma_{j l}+\beta_{l m} \sigma_{l j}\right)+\phi_{m l} \sigma_{j l}+\phi_{l m} \sigma_{l j}\right) \mathrm{d} V .
$$

\subsubsection{Dislocation part}

For the dislocation part of the system with the Lagrangian $\mathcal{L}_{\mathrm{di}}$ and $P_{j i}^{(\mathrm{d})}, \mathcal{P}_{j}^{(\mathrm{d})}$ given by (5.26) and (5.27) we get for the canonical currents

$$
\begin{aligned}
& M_{k i}^{(\mathrm{d})}:=\epsilon_{k m j}\left(x_{m} P_{j i}^{(\mathrm{d})}+D_{j i} \varphi_{m}-H_{j l i} \phi_{m l}-H_{l j i} \phi_{l m}\right) \\
& \mathcal{M}_{k}^{(\mathrm{d})}:=\epsilon_{k m j}\left(x_{m} \mathcal{P}_{j}^{(\mathrm{d})}+D_{j l} \phi_{m l}+D_{l j} \phi_{l m}\right) .
\end{aligned}
$$

Now the rotational balance law of the dislocation subsystem is given by

$$
\begin{aligned}
\mathrm{D}_{t} \mathcal{M}_{k}^{(\mathrm{d})}-\mathrm{D}_{i} M_{k i}^{(\mathrm{d})}=-\epsilon_{k m j}\left(x_{m} \mathcal{F}_{j}\right. & -\left(v_{m} p_{j}-\beta_{m l} \sigma_{j l}-\beta_{l m} \sigma_{l j}\right)+\dot{u}_{m} p_{j}+u_{m, l} \sigma_{j l}+u_{l, m} \sigma_{l j} \\
& \left.-I_{m l} D_{j l}-I_{l m} D_{l j}+\frac{1}{2} T_{m i l} H_{j i l}+T_{l i m} H_{l i j}\right) .
\end{aligned}
$$


The corresponding $\boldsymbol{L}$ integral reads

$$
\begin{aligned}
L_{k}^{(\mathrm{d})}= & \int_{S} M_{k i}^{(\mathrm{d})} n_{i} \mathrm{~d} S-\int_{V} \mathrm{D}_{t} \mathcal{M}_{k}^{(\mathrm{d})} \mathrm{d} V=-\int_{V} \epsilon_{k j m}\left(x_{m} \mathcal{F}_{j}-\left(v_{m} p_{j}-\beta_{m l} \sigma_{j l}-\beta_{l m} \sigma_{l j}\right)\right. \\
& \left.+\dot{u}_{m} p_{j}+u_{m, l} \sigma_{j l}+u_{l, m} \sigma_{l j}-I_{m l} D_{j l}-I_{l m} D_{l j}+\frac{1}{2} T_{m i l} H_{j i l}+T_{l i m} H_{l i j}\right) \mathrm{d} V .
\end{aligned}
$$

Finally, we get the decomposition of the $\boldsymbol{L}$ integral into the elastic and dislocation parts according

$$
L_{k}=L_{k}^{(\mathrm{e})}+L_{k}^{(\mathrm{d})}
$$

\section{Static case}

For the static case, the angular momentum tensor of the dislocation part is

$$
\begin{aligned}
M_{k i}^{(\mathrm{d})} & =\epsilon_{k m j}\left(x_{m} P_{j i}^{(\mathrm{d})}-H_{j l i} \phi_{m l}-H_{l j i} \phi_{l m}\right), \\
& =\epsilon_{k m j}\left(x_{m} P_{j i}^{(\mathrm{d})}-\epsilon_{l i n} H_{j n} \phi_{m l}\right)-\delta_{k i} H_{l n} \phi_{l n}+H_{l k} \phi_{l i}
\end{aligned}
$$

with the static $\boldsymbol{L}$ integrals as follows

$$
\begin{gathered}
L_{k}^{(\mathrm{d})}=\int_{S} M_{k i}^{(\mathrm{d})} n_{i} \mathrm{~d} S=\int_{V} \epsilon_{k j m}\left(x_{m} \mathcal{F}_{j}^{(\mathrm{Sta})}-\left(\beta_{m l} \sigma_{j l}+\beta_{l m} \sigma_{l j}\right)+u_{m, l} \sigma_{j l}+u_{l, m} \sigma_{l j}\right. \\
\left.+\frac{1}{2} T_{m i l} H_{j i l}+T_{l i m} H_{l i j}\right) \mathrm{d} V .
\end{gathered}
$$

Of course, for an isotropic material in a force stress-free state Eq. (5.57) is a conservation law.

\subsection{Scaling in space}

\subsubsection{Elastic part}

For the scaling we can also calculate for the Lagrangian $\mathcal{L}_{\mathrm{e}}$ the canonical currents

$$
\begin{aligned}
& Y_{i}^{(\mathrm{e})}:=x_{j} P_{j i}^{(\mathrm{e})}-t S_{i}^{(\mathrm{e})}+d_{u} \sigma_{j i} u_{j} \\
& \mathcal{Y}^{(\mathrm{e})}:=x_{j} \mathcal{P}_{j}^{(\mathrm{e})}-t \mathcal{H}^{(\mathrm{e})}+d_{u} p_{j} u_{j}
\end{aligned}
$$

where $P_{j i}^{(\mathrm{e})}, \mathcal{P}_{j}^{(\mathrm{e})}, S_{i}^{(\mathrm{e})}$ and $\mathcal{H}^{(\mathrm{e})}$ are given by (5.1)-(5.4). The corresponding balance law reads

$$
\mathrm{D}_{t} \mathcal{Y}^{(\mathrm{e})}-\mathrm{D}_{i} Y_{i}^{(\mathrm{e})}=-t \mathcal{W}+x_{k} \mathcal{F}_{k}+\frac{d}{2} p_{\alpha} \phi_{\alpha}-\frac{d}{2} \sigma_{\alpha \beta} \phi_{\alpha \beta}
$$

For the path-independent $M_{k}$ integral we obtain

$$
M^{(\mathrm{e})}:=\int_{S} Y_{i}^{(\mathrm{e})} n_{i} \mathrm{~d} S-\int_{V} \mathrm{D}_{t} \mathcal{Y}^{(\mathrm{e})} \mathrm{d} V=\int_{V}\left(t \mathcal{W}-x_{k} \mathcal{F}_{k}-\frac{d}{2} p_{\alpha} \phi_{\alpha}+\frac{d}{2} \sigma_{\alpha \beta} \phi_{\alpha \beta}\right) \mathrm{d} V
$$




\section{Static case}

In the static case, the scaling vector (5.58) is given in terms of the static Eshelby stress tensor (5.22) as follows

$$
Y_{i}^{(\mathrm{e})}=x_{j} P_{j i}^{(\mathrm{e})}+d_{u} \sigma_{j i} u_{j} .
$$

The static $M$ integral of the elastic subsystem reads

$$
M^{(\mathrm{e})}=\int_{S} Y_{i}^{(\mathrm{e})} n_{i} \mathrm{~d} S=-\int_{V}\left(x_{k} \mathcal{F}_{k}-\frac{d}{2} \sigma_{\alpha \beta} \phi_{\alpha \beta}\right) \mathrm{d} V .
$$

\subsubsection{Dislocation part}

For the canonical currents of the dislocation part from Eqs. (5.26)-(5.29) we get

$$
\begin{aligned}
& Y_{i}^{(\mathrm{d})}:=x_{j} P_{j i}^{(\mathrm{d})}-t S_{i}^{(\mathrm{d})}+d_{\varphi} D_{j i} \varphi_{j}-d_{\phi} H_{j l i} \phi_{j l}, \\
& \mathcal{Y}^{(\mathrm{d})}:=x_{j} \mathcal{P}_{j}^{(\mathrm{d})}-t \mathcal{H}^{(\mathrm{d})}+d_{\phi} D_{j l} \phi_{j l} .
\end{aligned}
$$

In general, the balance law derived from the dislocation part is given by

$$
\begin{aligned}
\mathrm{D}_{t} \mathcal{Y}^{(\mathrm{d})}-\mathrm{D}_{i} Y_{i}^{(\mathrm{d})}= & t \mathcal{W}-x_{k} \mathcal{F}_{k}+d_{\varphi} p_{\alpha} \varphi_{\alpha}-d_{\phi} \sigma_{\alpha \beta} \phi_{\alpha \beta}-\left(d_{\varphi}+\frac{d-2}{2}\right) D_{\alpha \beta} \varphi_{\alpha, \beta} \\
& +\left(d_{\phi}+\frac{d-2}{2}\right) D_{\alpha \beta} \dot{\phi}_{\alpha \beta}-\frac{1}{2}\left(d_{\phi}+\frac{d-2}{2}\right) H_{\alpha \beta k} T_{\alpha \beta k} .
\end{aligned}
$$

Using (4.58), the balance law (5.66) gets the form

$$
\mathrm{D}_{t} \mathcal{Y}^{(\mathrm{d})}-\mathrm{D}_{i} Y_{i}^{(\mathrm{d})}=t \mathcal{W}-x_{k} \mathcal{F}_{k}-\frac{d}{2} p_{\alpha} \varphi_{\alpha}+\frac{d}{2} \sigma_{\alpha \beta} \phi_{\alpha \beta}-2 \mathcal{L}_{\mathrm{di}}
$$

In integral form it reads

$$
M^{(1-\mathrm{d})}:=\int_{S} Y_{i}^{(\mathrm{d})} n_{i} \mathrm{~d} S-\int_{V} \mathrm{D}_{t} \mathcal{Y}^{(\mathrm{d})} \mathrm{d} V=-\int_{V}\left(t \mathcal{W}-x_{k} \mathcal{F}_{k}-\frac{d}{2} p_{\alpha} \phi_{\alpha}+\frac{d}{2} \sigma_{\alpha \beta} \phi_{\alpha \beta}-2 \mathcal{L}_{\mathrm{di}}\right) \mathrm{d} V .
$$

If we use (4.61), we obtain from (5.66) the following balance law

$$
\mathrm{D}_{t} \mathcal{Y}^{(\mathrm{d})}-\mathrm{D}_{i} Y_{i}^{(\mathrm{d})}=t \mathcal{W}-x_{k} \mathcal{F}_{k}-\frac{d-2}{2} p_{\alpha} \varphi_{\alpha}+\frac{d-2}{2} \sigma_{\alpha \beta} \phi_{\alpha \beta}
$$

For vanishing source terms of the Euler-Lagrange equations (2.21) and(2.22), the balance law (5.69) reduces to a conservation law. In integral form we have

$$
M^{(2-\mathrm{d})}:=\int_{S} Y_{i}^{(\mathrm{d})} n_{i} \mathrm{~d} S-\int_{V} \mathrm{D}_{t} \mathcal{Y}^{(\mathrm{d})} \mathrm{d} V=-\int_{V}\left(t \mathcal{W}-x_{k} \mathcal{F}_{k}-\frac{d-2}{2} p_{\alpha} \phi_{\alpha}+\frac{d-2}{2} \sigma_{\alpha \beta} \phi_{\alpha \beta}\right) \mathrm{d} V .
$$

Finally, the decomposition of the $M$ integral into the elastic and dislocation parts read

$$
\begin{aligned}
& M^{(1)}=M^{(\mathrm{e})}+M^{(1-\mathrm{d})}, \\
& M^{(2)}=M^{(\mathrm{e})}+M^{(2-\mathrm{d})} .
\end{aligned}
$$




\section{Static case}

In the static case, the scaling vector (5.64) is given in terms of the static Eshelby stress tensor (5.41) as follows

$$
Y_{i}^{(\mathrm{d})}=x_{j} P_{j i}^{(\mathrm{d})}+d_{\phi} H_{j l i} \phi_{j l}=x_{j} P_{j i}^{(\mathrm{d})}-d_{\phi} \epsilon_{i l m} H_{j m} \phi_{j l} .
$$

The static $M$ integrals of the dislocation subsystem with the choices (4.61) and (4.61) are given by

$$
\begin{aligned}
& M^{(1-\mathrm{d})}=\int_{S} Y_{i}^{(\mathrm{d})} n_{i} \mathrm{~d} S=\int_{V}\left(x_{k} \mathcal{F}_{k}^{\text {(Sta) }}-\frac{n}{2} \sigma_{\alpha \beta} \phi_{\alpha \beta}+2 W_{\mathrm{di}}\right) \mathrm{d} V, \\
& M^{(2-\mathrm{d})}=\int_{S} Y_{i}^{(\mathrm{d})} n_{i} \mathrm{~d} S=\int_{V}\left(x_{k} \mathcal{F}_{k}^{\text {(Sta) }}-\frac{n-2}{2} \sigma_{\alpha \beta} \phi_{\alpha \beta}\right) \mathrm{d} V .
\end{aligned}
$$

If $\sigma_{i j}=0$ (stress-free state), then only (5.75) is a conservation law.

\subsection{Gauge symmetry}

\subsubsection{Elastic part}

With the following currents

$$
\begin{aligned}
G_{i}^{(\mathrm{e})} & :=-\sigma_{j i} f_{j}, \\
\mathcal{G}^{(\mathrm{e})} & :=-p_{j} f_{j},
\end{aligned}
$$

we get

$$
\mathrm{D}_{t} \mathcal{G}^{(\mathrm{e})}-\mathrm{D}_{i} G_{i}^{(\mathrm{e})}=-p_{j} \dot{f}_{j}+\sigma_{j i} f_{j, i} .
$$

It is obvious that for $f_{i}=$ constant, Eq. (5.78) becomes a conservation law.

For the static case, we obtain

$$
\mathrm{D}_{i} G_{i}^{(\mathrm{e})}=-\sigma_{j i} f_{j, i}
$$

\subsubsection{Dislocation part}

For the dislocation part, the flux quantities are

$$
\begin{aligned}
G_{i}^{(\mathrm{d})} & :=D_{j i} \dot{f}_{j}-H_{j k i} f_{j, k}, \\
\mathcal{G}^{(\mathrm{d})} & :=D_{j i} f_{j, i},
\end{aligned}
$$

with the balance law

$$
\mathrm{D}_{t} \mathcal{G}^{(\mathrm{d})}-\mathrm{D}_{i} G_{i}^{(\mathrm{d})}=p_{j} \dot{f}_{j}-\sigma_{j i} f_{j, i} .
$$

If $f_{i}=$ constant, Eq. (5.82) becomes a conservation law.

For the static case, we get

$$
G_{i}^{(\mathrm{d})}=-H_{j k i} f_{j, k}=\epsilon_{i k l} H_{j l} f_{j, k},
$$


and the balance law

$$
\mathrm{D}_{i} G_{i}^{(\mathrm{d})}=\sigma_{j i} f_{j, i}
$$

For $\sigma_{j i}=0$ (stress-free state), the balance law (5.84) becomes a conservation law. This is the case for a pure dislocation theory without force stresses. If $f_{j, i}=$ constant, we recover the symmetry of constant pre-distortion used by Li et al. [38] and Li [39].

\subsection{Addition of solutions}

\subsubsection{Elastic part}

From Eqs. (4.74) and (4.75) we obtain for the elastic subsystem

$$
\begin{aligned}
& A_{i}^{(\mathrm{e})}=-\bar{u}_{\alpha} \sigma_{\alpha i}+u_{\alpha} \bar{\sigma}_{\alpha i}, \\
& A_{4}^{(\mathrm{e})}=\bar{u}_{\alpha} p_{\alpha}-u_{\alpha} \bar{p}_{\alpha} .
\end{aligned}
$$

They fulfill the corresponding balance law

$$
\mathrm{D}_{t} A_{4}^{(\mathrm{e})}+\mathrm{D}_{i} A_{i}^{(\mathrm{e})}=p_{\alpha} \dot{\bar{u}}_{\alpha}-\bar{p}_{\alpha} \dot{u}_{\alpha}-\sigma_{\alpha i} \bar{u}_{\alpha, i}+\bar{\sigma}_{\alpha i} u_{\alpha, i}
$$

For the static case we have the balance law

$$
\mathrm{D}_{i} A_{i}^{(\mathrm{e})}=-\sigma_{\alpha i} \bar{u}_{\alpha, i}+\bar{\sigma}_{\alpha i} u_{\alpha, i}
$$

\subsubsection{Dislocation part}

From Eqs. (4.74) and (4.75) we obtain for the dislocation subsystem

$$
\begin{aligned}
& A_{i}^{(\mathrm{d})}=-\bar{\varphi}_{\alpha} D_{\alpha i}+\bar{\phi}_{\alpha \beta} H_{\alpha \beta i}+\varphi_{\alpha} \bar{D}_{\alpha i}-\phi_{\alpha \beta} \bar{H}_{\alpha \beta i}, \\
& A_{4}^{(\mathrm{d})}=\bar{\phi}_{\alpha \beta} D_{\alpha \beta}-\phi_{\alpha \beta} \bar{D}_{\alpha \beta} .
\end{aligned}
$$

These quantities lead to the following balance law

$$
\mathrm{D}_{t} A_{4}^{(\mathrm{d})}+\mathrm{D}_{i} A_{i}^{(\mathrm{d})}=p_{\alpha} \bar{\varphi}_{\alpha}-\bar{p}_{\alpha} \varphi_{\alpha}-\sigma_{\alpha i} \bar{\phi}_{\alpha i}+\bar{\sigma}_{\alpha i} \phi_{\alpha i}
$$

If the source terms are zero $\left(p_{\alpha}=0, \bar{p}_{\alpha}=0, \sigma_{\alpha i}=0\right.$ and $\left.\bar{\sigma}_{\alpha i}=0\right)$ the balance law (5.91) becomes a conservation law.

In the static case we have

$$
A_{i}^{(\mathrm{d})}=\bar{\phi}_{\alpha \beta} H_{\alpha \beta i}-\phi_{\alpha \beta} \bar{H}_{\alpha \beta i}=\epsilon_{i \beta k}\left(\phi_{\alpha \beta} \bar{H}_{\alpha k}-\bar{\phi}_{\alpha \beta} H_{\alpha k}\right)
$$

with the balance law

$$
\mathrm{D}_{i} A_{i}^{(\mathrm{d})}=-\sigma_{\alpha i} \bar{\phi}_{\alpha i}+\bar{\sigma}_{\alpha i} \phi_{\alpha i}
$$

If $\sigma_{\alpha i}=0$ and $\bar{\sigma}_{\alpha i}=0$ (stress-free state), the balance law (5.93) becomes a conservation law. 


\section{Conclusion}

We have presented the equations of motion for the translational gauge field theory of dislocations with asymmetric stresses. For this purpose we have chosen the most general linear isotropic constitutive equations. The Lie-point symmetries leaving these system of equations form-invariant have been discussed. According to the Noether theorem, from the variational and divergence symmetries for the total Lagrangian density the currents in canonical form and the local conservation laws for the translational and rotational invariance have been derived. The form of the local balance law for the broken scaling symmetry has been found. The gauge-invariant currents have also been obtained. Taking into account only the elastic or dislocation part of the total Lagrangian density we have shown how the local conservation laws for the continuous transformation of translation and rotation turned into balance laws giving rise to a configurational force and moment. In this manner we have found the dynamical Peach-Koehler force. In addition we have calculated the configurational power for both parts. Since no external forces and moments act on the whole system described by the total Lagrangian the energy, linear and angular momentum are conserved. We also derived the conservation and balance laws for the gauge symmetry and the addition of solutions. Using the divergence symmetry of addition of solutions, we were able to derive a reciprocity theorem for the gauge theory of dislocations. In addition, we have derived the conservation laws for (force) stress-free states of dislocations. If we identify $\phi_{i j}=-\beta_{i j}^{\mathrm{P}}$ with the plastic distortion, our results and the formal structure of currents keep valid in gradient plasticity.

\section{Acknowledgement}

The authors have been supported by an Emmy-Noether grant of the Deutsche Forschungsgemeinschaft (Grant No. La1974/1-2).

\section{References}

[1] J.D. Eshelby, The force on an elastic singularity, Phil. Trans. Roy. Soc. London A 244, (1951) 87-112.

[2] J.D. Eshelby, The elastic energy-momentum tensor. J. Elasticity 5 (1975) 321-335.

[3] P.M. Morse, H. Feshbach, Methods of Theoretical Physics, McGraw-Hill, New York, 1953.

[4] W. Günther, Über einige Randintegrale der Elastostatik Abh. Braunschweig. Wiss. Ges. 14 (1962), 53-72.

[5] J.K. Knowles, E. Sternberg, On a class of conservation laws in linearized and finite elastostatics, Arch. Rational. Mech. Anal. 44 (1972) 187-211.

[6] D.C. Fletcher, Conservation laws in linear elastodynamics. Arch. Rational. Mech. Anal. 60 (1975), 329-353. 
[7] P.J. Olver, Conservation laws in elasticity. II. Linear homogeneous elastostatics, Arch. Rational. Mech. Anal. 85 (1984), 131-160; Errata in 102 (1988), 385-387.

[8] G.A. Maugin, Material Inhomogeneities in Elasticity. Chapman and Hall, London, 1993.

[9] R. Kienzler, G. Herrmann, Mechanics in Material Space. Berlin: Springer, 2000.

[10] L.D. Landau, E.M. Lifshitz, The classical theory of fields, fourth ed., Vol.2 (Course of theoretical Physics series), 1975.

[11] U.E. Schröder, Spezielle Relativitätstheorie, Verlag Harri Deutsch, Frankfurt am Main, Thun, 1994.

[12] A. Kadić, D.G.B. Edelen, A Yang-Mills type minimal coupling theory for materials with dislocations and disclinations, Int. J. Engng. Sci. 20 (1982) 433-438.

[13] A. Kadić, D.G.B. Edelen, A Gauge Theory of Dislocations and Disclinations, in: Lectures Notes in Physics, Vol. 174, Springer, Berlin (1983).

[14] D.G.B. Edelen and D.C. Lagoudas, Gauge theory and defects in solids, in: Mechanics and physics of discrete System, Vol. 1, G.C. Sih, ed., North-Holland, Amsterdam (1988).

[15] M.O. Peach, J.S. Koehler, Forces extended on dislocations and the stress fields produced by them, Phys. Rev. 80 (1950), 436-439.

[16] P.J. Olver, Applications of Lie groups to differential equations, Springer, New York, 1986.

[17] E. Cartan, On manifolds with an Affine Connection and the Theory of General Relativity (Engl. transl. of French original 1922/23) (Napoli: Bibliopolis).

[18] K. Kondo, On the geometrical and physical foundations of the theory of yielding. Proc.2. Japan nat. Congress of appl. Mech. (1952), 41-47.

[19] K. Kondo, Memoirs of the unifying study of the basic problems in engineering sciences by means of geometry. Vol. I, Tokyo: Gakujutsu Bunken Fukyu-Kai (1955).

[20] B.A. Bilby, R. Bullough and E. Smith, Continuous distributions of dislocations: a new application of the methods of non-Riemannian geometry. Proc. R. Soc. Lond. A 231, (1955), 263-273.

[21] B.A. Bilby, and E. Smith, Continuous distributions of dislocations. III, Proc. R. Soc. Lond. A 236, (1956), 481-505.

[22] E. Kröner, Kontinuumstheorie der Versetzungen und Eigenspannungen. Ergebn. Angew. Mathematik 5 (1958), 1-179.

[23] G.A. Maugin, On the structure of the theory of polar elasticity. Phil. Trans. R. Soc. Lond. A 356 (1998), 1367-1395. 
[24] E. Noether, Invariante Variationspropleme, Goettinger Nachrichten Mathematisch - Physikalische Klasse, 2 (1918), 235-256; (English translation: Invariant Variation Problems, 1971. Transport Theory and Stat. Phys. 1, 186-207).

[25] M. Lazar, On conservation and balance laws in micromorphic elastodynamics. J. Elast. 88 (2007), 63-78.

[26] V.A. Lubarda, X. Markenscoff, On conservation integrals in micropolar elasticity, Phil. Mag. 83 (2003), 1365-1377.

[27] B. Felsager, Geometry, Particles, and Fields. Springer, Berlin, 1998.

[28] I.S. Sokolnikoff, Mathematical Theory of Elasticity. McGraw-Hill Book Company, Inc, 1946.

[29] J.R. Willis, Dislocations and inclusions. J. Mech. Phys. Solids 13 (1985), 377-395.

[30] G. Kluge, Über den Zusammenhang der allgemeinen Versetzungstheorie mit dem Cosserat-Kontinuum. Wissenschaftliche Zeitschrift der Technischen Hochschule Otto von Guericke Magdeburg 13 (1969), 377-380.

[31] G. Kluge, Zur Dynamik der allgemeinen Versetzungstheorie bei Berücksichtigung von Momentenspannungen. Int. J. Engng. Sci. 7 (1969), 169-183.

[32] H. Schaefer, Maxwell-Gleichungen, Energiesatz und Lagrange-Dichte in der Kontinuumstheorie der Versetzungen. Acta Mech. 10 (1970), 59-66.

[33] E.F. Holländer, The geometric equation of dislocation dynamics, Czech. J. Phys. B 12 (1962), 35-47.

[34] H.O.K. Kirchner, The force on an elastic singularity in a nonhomogenous medium. J. Mech. Phys. Solids 47 (1999), 993-998.

[35] M. Lazar, H.O.K. Kirchner, The Eshelby stress tensor, angular momentum tensor and dilatation flux in gradient elasticity. Int. J. Solids Struct. 44 (2007), 2477-2486.

[36] M. Lazar, An elastoplastic theory of dislocations as a physical field theory with torsion. J. Phys. A: Math. Gen. 35 (2002), 1983-2004.

[37] M. Lazar, Screw dislocations in the field theory of elastoplasticity. Ann. Phys. (Leipzig) 11 (2002), 635-649.

[38] S. Li, C. Linder, J.W. Foulk III, On configurational compatibility and multiscale energy momentum tensors. J. Mech. Phys. Solids 55 (2007), 980-1000.

[39] S. Li, On variational symmetry of defect potentials and multiscale configurational force, Philosophical Magazine 88 (2008), 1059-1084. 